\title{
ODD SYMPLECTIC FLAG MANIFOLDS
}

\author{
ION ALEXANDRU MIHAI
}

\begin{abstract}
We define the odd symplectic grassmannians and flag manifolds, which are smooth projective varieties equipped with an action of the odd symplectic group and generalizing the usual symplectic grassmannians and flag manifolds. Contrary to the latter, which are the flag manifolds of the symplectic group, the varieties we introduce are not homogeneous. We argue nevertheless that in many respects the odd symplectic grassmannians and flag manifolds behave like homogeneous varieties; in support of this claim, we compute the automorphism group of the odd symplectic grassmannians, and we prove a Borel-Weil type theorem for the odd symplectic group.
\end{abstract}

\section{INTRODUCTION}

In Pro88 Proctor introduces the odd symplectic group, a generalization of the symplectic group on an odd dimensional space defined as the group of linear transformations preserving a generic skew-form. His initial motivation is a series of combinatorial identities, but Proctor goes on to study a certain class of representations of this group (which is not reductive) and eventually proves a character formula very similar to Weyl's formula for the simple Lie groups. In this way the odd symplectic group presents some similarities with the simple Lie groups and appears to fit nicely in the framework of the classical groups, filling the "gap" in the series $\left\{\mathrm{Sp}_{2 n}\right\}_{n}$.

Here, we take a different look at this situation, from a more geometric perspective. Recall that the flag manifolds of the symplectic group, $\mathrm{Sp}_{2 n} / P$, with $P$ a parabolic subgroup in $\mathrm{Sp}_{2 n}$, identify with the varieties of flags of isotropic subspaces of $\mathbb{C}^{2 n}$. We generalize these varieties to the odd symplectic situation, in the most straightforward way : let $\omega$ be a generic skew-form on $\mathbb{C}^{2 n+1}$ and define the odd symplectic flag manifolds to be the varieties of flags of subspaces of $\mathbb{C}^{2 n+1}$ isotropic with respect to $\omega$. These are projective varieties and are equipped with natural actions of the odd symplectic group $\mathrm{Sp}_{2 n+1}$ which preserves the skew-form $\omega$. Unlike in the symplectic setting however, these actions are no longer transitive, as the non trivial kernel of the skew-form is preserved by $\mathrm{Sp}_{2 n+1}$ and therefore isotropic flags having different incidence relations with this kernel cannot be in the same orbit.

The aim of this paper is to present some evidence that the odd symplectic flag manifolds, although not homogeneous, fill the role of flag manifolds for the odd symplectic group. Part of this evidence, for example, is constituted by the theorems below describing respectively the automorphism group of the odd symplectic grassmannians and a Borel-Weil theorem for the odd symplectic group, which show that sometimes the symplectic flag manifolds and their odd symplectic counterparts behave like a "series".

The reason we do not simply consider the homogeneous spaces $\mathrm{Sp}_{2 n+1} / P$, for $P \subset \mathrm{Sp}_{2 n+1}$ a parabolic subgroup, as "flag manifolds" for $\mathrm{Sp}_{2 n+1}$, is that, as it will become clear below, these coincide with the flag manifolds of the symplectic group $\mathrm{Sp}_{2 n}$, and therefore do not constitute representative examples for the odd symplectic situation. 
The odd symplectic flag manifolds are not homogeneous but it turns out they are quasi-homogeneous. The odd symplectic group acts with finitely many orbits, described by the incidence relations with the kernel mentioned above. They are also smooth, which follows from the fact that, as subvarieties of the (type A) flag manifolds defined by forgetting the isotropy conditions, they appear as the zero locus of a generic section of a vector bundle.

A natural question is whether the odd symplectic flag manifolds admit a cellular decomposition similar to the decomposition into Schubert cells of the usual symplectic flag manifolds. In the symplectic case, and in general for all the flag manifolds of the classical groups, the Schubert cells can be defined in several equivalent ways, eg they can be described by incidence relations with respect to a fixed flag and they coincide with the orbits of a Borel subgroup. It turns out that these two recipes can be used in the odd symplectic setting as well to define cell decompositions of the odd symplectic flag manifolds.

Actually, the odd symplectic flag manifolds themselves can be identified with certain Schubert subvarieties in symplectic flag manifolds (and then the cell decompositions above coincide with their cell decomposition as Schubert varieties). This goes as follows: the generic skew-form $\omega$ on $\mathbb{C}^{2 n+1}$ can be extended to a symplectic form $\tilde{\omega}$ on $\mathbb{C}^{2 n+2}$, so that any odd symplectic flag manifold associated to $\omega$ is identified with the Schubert subvariety of the corresponding symplectic flag manifold associated to $\tilde{\omega}$ given by those flags which are contained in the hyperplane $\mathbb{C}^{2 n+1}$. The parabolic subgroup of $\mathrm{Sp}_{2 n+2}$ which preserves the hyperplane $\mathbb{C}^{2 n+1}$ acts therefore on the odd symplectic flag manifolds, via the morphism of restriction to $\mathbb{C}^{2 n+1}$ which is surjective with image $\mathrm{Sp}_{2 n+1}$.

The subgroup $\widetilde{\mathrm{Sp}}_{2 n+1}$ of this parabolic subgroup which fixes an equation of the hyperplane $\mathbb{C}^{2 n+1}$ (or, equivalently, a vector $e$ generating the kernel of $\omega$ ) has been considered before by Gelfand and Zelevinski in GZ84 as a variant odd symplectic group, in connection to the problem of constructing representation models for the classical groups. We will call it the intermediate odd symplectic group to distinguish it from the odd symplectic group considered above. This group has been considered later by Shtepin in Sht93 where he constructs a series of $\widetilde{\mathrm{Sp}}_{2 n+1}$-modules as a means to separate multiple components when restricting simple $\mathrm{Sp}_{2 n+2}$-modules to $\mathrm{Sp}_{2 n}$. Its Lie algebra $\widetilde{\mathfrak{s p}}_{2 n+1}$ coincides with the intermediate Lie algebra of the symplectic Lie algebra $\mathfrak{s p}_{2 n+2}$, a general construction which can be associated to any simple Lie algebra (which is used for example in [LM06] to construct the Lie algebra $\mathfrak{e}_{7 \frac{1}{2}}$ sitting between the exceptional Lie algebras $\mathfrak{e}_{7}$ and $\mathfrak{e}_{8}$ ).

In this paper we will focus only on the extremal types of odd symplectic flag manifolds, namely the odd symplectic grassmannians

$$
G_{\omega}(k, 2 n+1)=\left\{V \mid V \subset \mathbb{C}^{2 n+1}, \operatorname{dim} V=k, V \text { isotropic } / \omega\right\}
$$

and the variety of maximal flags of isotropic subspaces $\mathbb{F}_{\omega}(2 n+1)$, which we simply call the odd symplectic flag manifold. Since the maximal isotropic subspaces in $\mathbb{C}^{2 n+1}$ are those of dimension $n+1$, the variety $\mathbb{F}_{\omega}(2 n+1)$ is the variety of flags of the form $V_{\bullet}=\left(V_{1} \subset V_{2} \subset \cdots \subset V_{n+1}\right)$ with each $V_{i} \subset \mathbb{C}^{2 n+1}$ isotropic of dimension $i$. These varieties are the analogues in the odd symplectic setting of the symplectic grassmannians $G_{\omega}(k, 2 n)$ and the odd symplectic flag manifold $\mathbb{F}_{\omega}(2 n)$, which are, respectively, the minimal and the maximal flag varieties of the symplectic group $\mathrm{Sp}_{2 n}$.

We compute the automorphism group of the odd symplectic grassmannians $G_{\omega}(k, 2 n+1)$ and find out that for $2 \leqslant k \leqslant n$ it equals $\operatorname{PSp}_{2 n+1}$, the quotient of $\operatorname{Sp}_{2 n+1}$ by its center $\{ \pm 1\}$ (for $k=n+1$ the odd symplectic grassmannian $G_{\omega}(n+1,2 n+1)$ is isomorphic to the symplectic grassmannian $G_{\omega}(n, 2 n)$ and 
therefore its automorphism group is $\left.\mathrm{PSp}_{2 n}\right)$. In this respect the odd symplectic grassmannians behave like homogeneous flag manifolds, since, as a general rule, the connected automorphism group $\operatorname{Aut}^{\circ}(G / P)$ of a flag manifold is the adjoint group $\mathrm{P} G$ (there are some exceptions to this rule, see Akh95 §3.3, Theorem 2]) . This is also suggestive of the behavior of the symplectic and odd symplectic grassmannians as a series, as we can state this result in the following uniform manner:

Theorem. For integers $N$ and $k$ such that $2 \leqslant k \leqslant[N / 2]$, the automorphism group of the variety $G_{\omega}(k, N)$ is $\operatorname{PSp}_{N}=\operatorname{Sp}_{N} /\{ \pm 1\}$.

Finally, this also shows a close connection between the group $\mathrm{Sp}_{2 n+1}$ and the odd symplectic grassmannians $G_{\omega}(k, 2 n+1)$ since, up to the center $\{ \pm 1\}$, we can recover $\mathrm{Sp}_{2 n+1}$ from the geometry of $G_{\omega}(k, 2 n+1$ ) (which is not the case for the homogeneous spaces $\mathrm{Sp}_{2 n+1} / P, P$ a parabolic subgroup of $\mathrm{Sp}_{2 n+1}$ ).

Another natural question is whether there is an analog of the Borel-Weil theorem for the odd symplectic group. The usual Borel-Weil theorem connects the representation theory of a simple complex Lie group $G$ with the geometry of the flag variety $G / B$ by explicitly identifying the simple $G$-modules with the spaces of global sections of the line bundles on $G / B$. There are two points that need to be addressed in order to generalize this to the odd symplectic setting. First, the odd symplectic group is not reductive and therefore the simple modules do not play the same role in its representation theory as they do in the symplectic case. We need to replace them by another class of preferred representations, and it is natural to consider the class of $\mathrm{Sp}_{2 n+1}$-modules introduced by Proctor. These are defined by porting to the odd symplectic setting the construction of Weyl of the simple modules of the symplectic group. Specifically, for $\lambda$ a partition with at most $n+1$ parts define the $\mathrm{Sp}_{2 n+1}$-module $S_{\langle\lambda\rangle} \mathbb{C}^{2 n+1}$ as the intersection of the Schur power $S_{\lambda} \mathbb{C}^{2 n+1}$ with the kernels of all the possible contractions with the odd symplectic form $\omega$ (the "trace free" part of $S_{\lambda} \mathbb{C}^{2 n+1}$ ). We may also consider the representations of the intermediate odd symplectic group $\widetilde{\mathrm{Sp}}_{2 n+1}$ which were introduced by Shtepin. We show that actually these are isomorphic to the representations defined by Proctor, via the natural morphism $\widetilde{\mathrm{Sp}}_{2 n+1} \rightarrow \mathrm{Sp}_{2 n+1}$. The second point that needs attention is that the odd symplectic flag manifold $\mathbb{F}_{\omega}(2 n+1)$ is not homogeneous, so we no longer have a correspondence between line bundles and characters as in the symplectic case. It is natural then to use the fact that $\mathbb{F}_{\omega}(2 n+1)$ identifies with a Schubert subvariety in the symplectic flag manifold $\mathbb{F}_{\omega}(2 n+2)$ and consider those line bundles on $\mathbb{F}_{\omega}(2 n+1)$ which come from $\mathbb{F}_{\omega}(2 n+2)$. We write them in terms of the tautological bundles, and eventually obtain:

Theorem. For $N$ an integer let $n=[(N-1) / 2]$. Let $\lambda=\left(\lambda_{0} \geqslant \lambda_{1} \geqslant \cdots \geqslant \lambda_{n}\right)$ be a partition with at most $n+1$ parts. Denote by $L_{\lambda}$ the line bundle on $\mathbb{F}_{\omega}\left(\mathbb{C}^{N}\right)$ given by

$$
L_{\lambda}=T_{1}^{* \otimes \lambda_{0}} \otimes\left(T_{2} / T_{1}\right)^{* \otimes \lambda_{1}} \otimes \cdots \otimes\left(T_{n+1} / T_{n}\right)^{* \otimes \lambda_{n}}
$$

where $T_{i}$ is the rank $i$ tautological vector bundle on $\mathbb{F}_{\omega}\left(\mathbb{C}^{N}\right)$. Then, as $\mathrm{Sp}_{N}$-modules, we have

$$
H^{0}\left(\mathbb{F}_{\omega}\left(\mathbb{C}^{N}\right), L_{\lambda}\right) \simeq\left(S_{\langle\lambda\rangle} \mathbb{C}^{N}\right)^{*} .
$$

Here, when $N=2 n+2$ we get the usual Borel-Weil theorem for the symplectic group $\mathrm{Sp}_{2 n+2}$. So again we notice the behavior of the symplectic and odd symplectic flag manifolds as a series.

In a forthcoming paper ( Mih $)$ we study the equivariant cohomology of the odd symplectic flag manifold $\mathbb{F}_{\omega}(2 n+1)$ correspondig to the action of a maximal torus of $\mathrm{Sp}_{2 n+1}$. In particular, we compute the singular cohomology algebra $H^{*}\left(\mathbb{F}_{\omega}(2 n+\right.$ 
$1), \mathbb{C})$ which turns out to be isomorphic to the singular cohomology algebra of the flag manifold $G / B$ for $G$ of type $\mathrm{D}_{n+1}$.

Overview. This paper is organized as follows. In the second section we gather some basic facts and fix notation concerning the symplectic groups and their flag manifolds. In the third section we introduce Proctor's odd symplectic group and list some of its properties. We also recall here the intermediate odd symplectic group of Gelfand and Zelevinski and its relation with the odd symplectic group. In the fourth section we define the odd symplectic flag manifolds. We describe the $\mathrm{Sp}_{2 n+1}$-orbits and the Schubert decompositions in the case of the odd symplectic grassmannians and the odd symplectic flag manifold. The fifth section deals with the computation of the automorphism group of the odd symplectic grassmannians. We conclude, in the sixth section, with the Borel-Weil theorem for the odd symplectic group.

Acknowledgements. I warmly thank Laurent Manivel for his invaluable help and support in writing this article. I also thank Patrick Polo for his very useful comments concerning section 6 . This paper has been written while I was a post-doc fellow at the Weizmann Institute of Science under the Marie Curie RTN Liegrits. I wish to thank my hosts for their hospitality and the perfect working conditions they provided.

\section{Contents}

\begin{tabular}{|c|}
\hline 1. Introduction \\
\hline 2. Preliminaries \\
\hline 3. The odd svmplectic group \\
\hline 4. Odd svmplectic flag manifolds \\
\hline 5. The automorphism group of an odd svmplectic grassmannian \\
\hline 6. A Borel-Weil theorem for the odd svmplectic group \\
\hline References \\
\hline
\end{tabular}

\section{Preliminaries}

We start by reviewing some basic facts about the symplectic group and its flag manifolds.

2.1. Let $F$ complex vector space of dimension $2 n$ and $\omega \in \Lambda^{2} F^{*}$ a symplectic form on $F$, ie a skew-symmetric, non-degenerate form. A subspace $V \subset F$ is isotropic if $\omega(x, y)=0$ for all $x, y \in V$. A symplectic basis is a basis $\left\{e_{1}, \ldots, e_{2 n}\right\}$ of $F$ such that

$$
\omega\left(e_{i}, e_{j}\right)=\delta_{i, 2 n+1-j}
$$

for all $1 \leqslant i, j \leqslant 2 n$. Any symplectic form admits a symplectic basis. A standard notation convention, which we will also use in this paper, is to denote $\bar{\imath}=2 n+1-i$ for $i \in\{1, \ldots, 2 n\}$. A symplectic basis is therefore a basis $\left\{e_{1}, \ldots, e_{n}, e_{\bar{n}}, \ldots, e_{\overline{1}}\right\}$ such that $\omega\left(e_{i}, e_{\bar{\jmath}}\right)=\delta_{i j}$ for all $1 \leqslant i, j \leqslant 2 n$. In a symplectic basis, the matrix of the form $\omega$ is

$$
J=\left(\begin{array}{cc}
0 & A \\
-A & 0
\end{array}\right)
$$

where $A$ is the $n \times n$ matrix whose anti-diagonal entries are all equal to 1 and all the other entries are 0 . 
2.2. The symplectic group $\mathrm{Sp}_{2 n}$ is the stabilizer of $\omega$ in $\mathrm{GL}(F)$ for the standard action of $\mathrm{GL}(F)$ in $\Lambda^{2} F^{*}$. It is a simple algebraic group. The choice of a symplectic basis identifies $\mathrm{Sp}_{2 n}$ with the matrix group

$$
\left\{g \in \mathrm{GL}(2 n, \mathbb{C}) \mid{ }^{t} g J g=J\right\} .
$$

From now on, we fix a symplectic basis $\left\{e_{1}, \ldots, e_{2 n}\right\}$ and identify $\operatorname{Sp}_{2 n}$ with this matrix group.

The subgroup of $\mathrm{Sp}_{2 n}$ of upper triangular matrices in the basis $\left\{e_{1}, \ldots, e_{2 n}\right\}$ is a Borel subgroup. Also, the subgroup $T_{2 n}$ of $\mathrm{Sp}_{2 n}$ of diagonal matrices in the basis $\left\{e_{1}, \ldots, e_{2 n}\right\}$ is a maximal torus. We have

$$
T_{2 n}=\left\{\operatorname{diag}\left(t_{1}, \ldots, t_{n}, t_{n}^{-1}, \ldots, t_{1}^{-1}\right) \mid t_{1}, \ldots, t_{n} \in \mathbb{C}^{*}\right\},
$$

where $\operatorname{diag}\left(x_{1}, \ldots, x_{2 n}\right)$ denotes the diagonal matrix whose diagonal entries are $x_{1}, \ldots, x_{2 n}$. Denote by $\varepsilon_{1}, \ldots, \varepsilon_{2 n}$ the characters $\varepsilon_{i}: \operatorname{diag}\left(x_{1}, \ldots, x_{2 n}\right) \mapsto x_{i}$ of $T_{2 n}$. Then

$$
\varepsilon_{\bar{\imath}}=-\varepsilon_{i}
$$

for all $1 \leqslant i \leqslant n$ and the characters $\varepsilon_{1}, \ldots, \varepsilon_{n}$ form a basis of the character group of $T_{2 n}$.

2.3. The Lie algebra of the symplectic group identifies with the subalgebra of $\mathfrak{g l}_{2 n}$

$$
\mathfrak{s p}_{2 n}=\left\{X \in \mathfrak{g l}_{2 n} \mid{ }^{t} X J+J X=0\right\} .
$$

We have $X=\left(x_{i j}\right) \in \mathfrak{s p}_{2 n}$ if and only if

$$
x_{i j}=-x_{\bar{\jmath}}, x_{i \bar{\jmath}}=x_{j \bar{\imath}}, x_{\bar{\imath} j}=x_{\bar{\jmath} i}
$$

for all $1 \leqslant i, j \leqslant n$. Let $E_{i j}$ by the elementary matrix with a 1 in the $i$ row and $j$ column, and denote

$$
\begin{aligned}
& X_{i j}=E_{i j}-E_{\bar{\jmath}} \text { for } 1 \leqslant i, j \leqslant n \text { and } \\
& X_{i \bar{\jmath}}=E_{i \bar{\jmath}}+E_{j \bar{\imath}}, \quad X_{\bar{\imath} j}=E_{\bar{\imath} j}+E_{\bar{\jmath} i}, \text { for } 1 \leqslant i \leqslant j \leqslant n .
\end{aligned}
$$

These elements make a basis of $\mathfrak{s p}_{2 n}$.

The elements $X_{i i}, 1 \leqslant i \leqslant n$, form a basis of the Cartan subalgebra $\mathfrak{t}_{2 n}$ of diagonal matrices in $\mathfrak{s p}_{2 n}$. The elements $X_{i j}, X_{i \bar{\jmath}}, 1 \leqslant i \leqslant j \leqslant n$, form a basis of the Borel subalgebra $\mathfrak{b}_{2 n}$ of $\mathfrak{s p}_{2 n}$ of upper triangular matrices. The roots of $\mathfrak{s p}_{2 n}$ are

$$
\begin{array}{ll} 
\pm\left(\varepsilon_{i}-\varepsilon_{j}\right), & 1 \leqslant i<j \leqslant n \\
\pm\left(\varepsilon_{i}+\varepsilon_{j}\right), & 1 \leqslant i \leqslant j \leqslant n
\end{array}
$$

and for $1 \leqslant k \neq \ell \leqslant 2 n$, the root space of $\mathfrak{s p}_{2 n}$ corresponding to the root $\varepsilon_{k}-\varepsilon_{\ell}$ is $\mathbb{C} X_{k \ell}$. In particular, the roots of the Borel subalgebra $\mathfrak{b}_{2 n}$, which we choose as positive roots, are $\varepsilon_{i}-\varepsilon_{j}, 1 \leqslant i<j \leqslant n$, and $\varepsilon_{i}+\varepsilon_{j}, 1 \leqslant i \leqslant j \leqslant n$.

2.4. The Weyl group $W\left(\operatorname{Sp}_{2 n}\right)=N_{\mathrm{Sp}_{2 n}}\left(T_{2 n}\right) / T_{2 n}$ of the symplectic group $\mathrm{Sp}_{2 n}$ is isomorphic to the group of linear transformations of $F$ which permute the vectors $e_{i}$ of the symplectic basis and commute with the matrix $J$. It identifies with the group of permutations of the set $\{1, \ldots, 2 n\}$ which commute with the involution $i \mapsto \bar{\imath}$

$$
W\left(\mathrm{Sp}_{2 n}\right)=\left\{w \in S_{2 n} \mid w(\bar{\imath})=\overline{w(i)}\right\},
$$

the correspondence being given by

$$
w \cdot e_{i}=e_{w(i)}, \quad 1 \leqslant i \leqslant 2 n, w \in W\left(\mathrm{Sp}_{2 n}\right) .
$$

Then the action of $W\left(\mathrm{Sp}_{2 n}\right)$ on the character group of the maximal torus is given by the rule

$$
w \cdot \varepsilon_{i}=\varepsilon_{w(i)}, \quad 1 \leqslant i \leqslant n, w \in W\left(\operatorname{Sp}_{2 n}\right) .
$$


Any element $w \in W\left(\mathrm{Sp}_{2 n}\right)$ is determined by its values on the set $\{1, \ldots, n\}$. To represent $w$ we use the one-line notation $w(1) w(2) \ldots w(n)$ with the convention to write $\bar{\imath}$ instead of $j=\bar{\imath}$ when $j \geqslant n+1$. For example, for $n=4,1674$ gets written $1 \overline{3} \overline{2} 4$. Given that $\varepsilon_{\bar{\imath}}=-\varepsilon_{i}$, the bars in the one-line notation correspond to sign changes in the action on the characters, and $W\left(\mathrm{Sp}_{2 n}\right)$ identifies in this way to the signed permutations of the $\varepsilon_{1}, \ldots, \varepsilon_{n}$.

The group $W\left(\mathrm{Sp}_{2 n}\right)$ is a Coxeter group generated by the reflections corresponding to the simple roots. The length $\ell(w)$ of an element $w$ is the minimal number of simple reflections required to express $w$ as a product of reflections. The longest element of $W\left(\mathrm{Sp}_{2 n}\right)$ is $\overline{1} \overline{2} \ldots \bar{n}$ and its length is $n^{2}$.

The Bruhat order on the Weyl group $W\left(\mathrm{Sp}_{2 n}\right)$ is defined by $w \leqslant w^{\prime}$ if and only if there is a chain

$$
w=w_{1} \rightarrow w_{2} \rightarrow \cdots \rightarrow w_{d}=w^{\prime}
$$

such that, for all $1 \leqslant i \leqslant d-1, \ell\left(w_{i+1}\right)=\ell\left(w_{i}\right)+1$ and $w_{i+1}=\sigma_{\alpha} w_{i}$ for a root $\alpha$.

2.5. Symplectic flag manifolds. For $1 \leqslant d_{1}<d_{2}<\cdots<d_{r} \leqslant n$ a sequence of integers, denote by $\mathbb{F}_{\omega}\left(d_{1}, \ldots, d_{r}, F\right)$ the projective variety of flags of isotropic subspaces

$$
\left\{\left(V_{d_{1}} \subset \cdots \subset V_{d_{r}} \subset F\right) \mid \operatorname{dim} V_{d_{i}}=d_{i}, V_{d_{i}} \text { isotropic for all } 1 \leqslant i \leqslant r\right\} .
$$

The symplectic group acts naturally on this variety and the action is transitive, ie this variety is a homogeneous space $\mathrm{Sp}_{2 n} / P$ with $P$ a parabolic subgroup. Conversely, any homogeneous space $\mathrm{Sp}_{2 n} / P$ with $P$ a parabolic subgroup is isomorphic to one of the varieties $\mathbb{F}_{\omega}\left(d_{1}, \ldots, d_{r}, F\right)$. We call them symplectic flag manifolds.

When $r=1$ we will write $G_{\omega}(k, F)$ instead of $\mathbb{F}_{\omega}(k, F)$ and call the varieties $G_{\omega}(k, F)$ symplectic grassmannians. These are subvarieties of the usual grassmannians $G(k, F)$ and are isomorphic to the homogeneous spaces $\mathrm{Sp}_{2 n} / P$ with $P$ a maximal parabolic subgroup. When $r=n$ we denote $\mathbb{F}_{\omega}(F)=\mathbb{F}_{\omega}(1, \ldots, n, F)$ and, if there is no risk of confusion, simply call $\mathbb{F}_{\omega}(F)$ the symplectic flag manifold. This is isomorphic to the homogeneous space $\mathrm{Sp}_{2 n} / B$ with $B$ a Borel subgroup. Since the isomorphism class of these varieties depends only on the dimension of $F$, we will also write $G_{\omega}(k, 2 n)$ and $\mathbb{F}_{\omega}(2 n)$ instead of $G_{\omega}(k, F)$ and, respectively, $\mathbb{F}_{\omega}(F)$.

2.6. Schubert cells in symplectic grassmannians. Let's fix in $F$ the flag $E_{\bullet}=\left(E_{1} \subset E_{2} \subset \cdots \subset E_{2 n}\right)$ generated by the symplectic basis $\left\{e_{1}, \ldots, e_{2 n}\right\}$ ie such that $E_{i}=\left\langle e_{1}, \ldots, e_{i}\right\rangle$ for all $1 \leqslant i \leqslant 2 n$ (we also say that $\left\{e_{1}, \ldots, e_{2 n}\right\}$ is an adapted basis for the flag $\left.E_{\bullet}\right)$.

2.7. The incidence of a $k$-dimensional subspace $V \subset F$ with the flag $E_{\bullet}$ is given by the integers $r_{i}=\operatorname{dim}\left(V \cap E_{i}\right)$. Let $i_{1}<i_{2}<\cdots<i_{k}$ be the positions of the $k$ jumps in the sequence

$$
0=r_{0} \leqslant r_{1} \leqslant \cdots \leqslant r_{2 n-1} \leqslant r_{2 n}=k
$$

that is

$$
i_{\alpha}=\min \left\{i \mid 1 \leqslant i \leqslant 2 n, r_{i}=\alpha\right\}, \quad \text { for } 1 \leqslant \alpha \leqslant k .
$$

We say that the multi-index $I=\left(1 \leqslant i_{1}<i_{2}<\cdots<i_{k} \leqslant 2 n\right)$ is the incidence type of $V$ with the flag $E_{\bullet}$.

Not all indices appear as incidence types of isotropic subspaces. We call those who do admissible. These are precisely those $I=\left(1 \leqslant i_{1}<\cdots<i_{k} \leqslant 2 n\right)$ for which $i_{\alpha} \neq \overline{i_{\beta}}$ for all $1 \leqslant \alpha, \beta \leqslant k$, that is, for each $1 \leqslant i \leqslant n$, at most one of $i$ or $\bar{\imath}$ appears in $I$.

Notation 2.8. We denote $\mathbf{I}_{k, 2 n}$ the set of multi-indices $\left(1 \leqslant i_{1}<i_{2}<\cdots<i_{k} \leqslant\right.$ $2 n)$ and $\mathbf{I}_{k, 2 n}^{\omega}$ the subset of $\mathbf{I}_{k, 2 n}$ of admissible indices. 
The Schubert cell in the symplectic grassmannian $G_{\omega}(k, 2 n)$ associated (with respect to the flag $E_{\bullet}$ ) to an admissible multi-index $I \in \mathbf{I}_{k, 2 n}^{\omega}$ is the set of isotropic subspaces of dimension $k$ whose incidence type is $I$, ie

$$
C_{I}=\left\{V \in G_{\omega}(k, 2 n) \mid \operatorname{dim}\left(V \cap E_{i}\right)=\alpha, \quad i_{\alpha} \leqslant i<i_{\alpha+1}, 1 \leqslant \alpha \leqslant k\right\},
$$

where we put $i_{k+1}=2 n+1$. If $B_{2 n}$ denotes the Borel subgroup of $\mathrm{Sp}_{2 n}$ which fixes the flag $E_{\bullet}$, then the Schubert cells, which are clearly $B_{2 n}$-stable, are actually $B_{2 n}$-orbits. The symplectic grassmannian $G_{\omega}(k, F)$ decomposes as a disjoint union

$$
G_{\omega}(k, F)=\bigcup_{I \in \mathbf{I}_{k, 2 n}^{\omega}} C_{I} .
$$

2.9. The Schubert cell $C_{I}$ is the set of isotropic subspaces of dimension $k$ which, with respect to the symplectic basis $\left\{e_{1}, \ldots, e_{2 n}\right\}$, can be generated by the rows of a row-echelon matrix of type $I$, that is a $k \times 2 n$ matrix $\left(a_{\alpha i}\right)$ for which each entry $a_{\alpha i_{\alpha}}$ is non zero and all the entries to its right are zero. Each $V \in \mathbb{C}_{I}$ is generated by the rows of a unique such matrix if we require additionally that the entries $a_{\alpha i_{\alpha}}$ be 1 and the entries below an entry $a_{\alpha i_{\alpha}}$ be zero. The remaining entries are free entries except for those below an entry opposed to a 1 (ie below an entry $a_{\alpha \overline{i_{\alpha}}}$ ) which are determined. The free entries define an isomorphism between $C_{I}$ and the affine space $\mathbb{A}^{\operatorname{dim} C_{I}}$.

For example, in the symplectic grassmannian $G_{\omega}(3,8)$ the Schubert cell defined by the multi-index $(4,6,8)=(4, \overline{3}, \overline{1})$ is given by the row-space of the matrix

$$
\left(\begin{array}{llllllll}
* & * & * & 1 & 0 & 0 & 0 & 0 \\
* & * & * & 0 & \bullet & 1 & 0 & 0 \\
* & * & \bullet & 0 & \bullet & 0 & * & 1
\end{array}\right)
$$

We have marked by $*$ the free entries and by $\bullet$ those which are determined.

2.10. On the set $\mathbf{I}_{k, 2 n}$ we consider the order relation for which $I \leqslant J$ if and only if $i_{\alpha} \leqslant j_{\alpha}$ for all $1 \leqslant \alpha \leqslant k$. We consider the induced order on the subset $\mathbf{I}_{k, 2 n}^{\omega} \subset \mathbf{I}_{k, 2 n}$ of admissible indices.

Let $P_{k}$ be the parabolic subgroup of $\mathrm{Sp}_{2 n}$ which stabilizes the subspace $E_{k}=$ $\left\langle e_{1}, \ldots, e_{k}\right\rangle$, so that $G_{\omega}(k, F)=\mathrm{Sp}_{2 n} / P_{k}$. Then $P_{k}$ is the maximal parabolic subgroup which misses the simple root $\alpha_{k}=\varepsilon_{k}-\varepsilon_{k+1}$. The Weyl group $W\left(P_{k}\right)$ of $P_{k}$ is isomorphic to $S_{k} \times W\left(\mathrm{Sp}_{2(n-k)}\right)$. The coset space $W\left(\mathrm{Sp}_{2 n}\right) / W\left(P_{k}\right)$ is in bijection with the $T_{2 n}$-fixed points in $\mathrm{Sp}_{2 n} / P_{k}$, and therefore with $\mathbf{I}_{k, 2 n}^{\omega}$. Explicitly, this bijection associates to the class of $w \in W\left(\operatorname{Sp}_{2 n}\right)$ the multi-index $\{w(1), \ldots, w(k)\} \uparrow$ obtained by arranging in increasing order the elements of the set $\{w(1), \ldots, w(k)\}$. Via this bijection, the order on $\mathbf{I}_{k, 2 n}^{\omega}$ corresponds with the order on $W\left(\operatorname{Sp}_{2 n}\right) / W\left(P_{k}\right)$ inherited from the Bruhat order on $W\left(\mathrm{Sp}_{2 n}\right)$, and so with the Bruhat order on the set of Schubert cells. That is, for all $I, J \in \mathbf{I}_{k, 2 n}^{\omega}$,

$$
C_{I} \subset \overline{C_{J}} \Longleftrightarrow I \leqslant J .
$$

2.11. The Schubert subvarieties of the symplectic grassmannian $G_{\omega}(k, F)$ are the closures of the Schubert cells. Since the Bruhat order of the Schubert cells agrees with the order on $\mathbf{I}_{k, 2 n}^{\omega}$, the Schubert subvariety $X_{I}=\overline{C_{I}}$ is given by

$$
X_{I}=\bigcup_{J \leqslant I} C_{J}
$$

Therefore, in terms of the incidence with the flag $E_{\bullet}$, the Schubert subvariety $X_{I}$ is characterized by

$$
X_{I}=\left\{V \in G_{\omega}(k, F) \mid \operatorname{dim}\left(V \cap E_{i_{\alpha}}\right) \geqslant \alpha, \quad 1 \leqslant \alpha \leqslant k\right\} .
$$


2.12. Schubert cells in the symplectic flag manifold. For a signed permutation $w \in W\left(\mathrm{Sp}_{2 n}\right)$ the rank function $r_{w}$ is the function which to a pair $(i, j) \in\{1, \ldots, n\} \times\{1, \ldots, 2 n\}$ associates the number

$$
r_{w}(i, j)=\operatorname{card}\{k \mid k \leqslant i, w(k) \leqslant j\} .
$$

The integer $r_{w}(i, j)$ is therefore the number of points in the graph $\{(k, w(k)) \mid 1 \leqslant$ $k \leqslant n\}$ of $w$, situated in the rectangle $\{1, \ldots, i\} \times\{1, \ldots, j\}$.

The Schubert cell $C_{w}$ of the symplectic flag manifold $\mathbb{F}_{\omega}(F)$ associated (with respect to the flag $\left.E_{\bullet}\right)$ to a signed permutation $w \in W\left(\mathrm{Sp}_{2 n}\right)$ is the set of flags of isotropic subspaces $V_{\bullet}=\left(V_{1} \subset \cdots \subset V_{n}\right)$ which verify

$$
\operatorname{dim}\left(V_{i} \cap E_{j}\right)=r_{w}(i, j)
$$

for all $1 \leqslant i \leqslant n$ and all $1 \leqslant j \leqslant 2 n$. The Schubert cells coincide with the orbits of the Borel subgroup $B_{2 n}$ of $\mathrm{Sp}_{2 n}$ which fixes the flag $E_{\bullet}$. Note that we have $V_{\bullet} \in C_{w}$ if and only if $V_{i} \in C_{\{w(1), \ldots, w(i)\} \uparrow}$ for all $1 \leqslant i \leqslant n$.

2.13. The Schubert cell $C_{w}$ is the set of flags of isotropic subspaces which, with respect to the symplectic basis $\left\{e_{1}, \ldots, e_{2 n}\right\}$, admit as adapted basis the rows of a $n \times 2 n$ matrix $\left(a_{i j}\right)$ for which $a_{i w(i)}=1$ and $a_{i j}=0$ for all $1 \leqslant i \leqslant n$ and $1 \leqslant j \leqslant 2 n, j>w(i)$. Note that the entries which are preassigned the value 1 correspond to the points of the graph of $w$.

2.14. The Bruhat order on $W\left(\mathrm{Sp}_{2 n}\right)$ agrees with the Bruhat order on the set of Schubert cells, that is, for all $w, w^{\prime} \in W\left(\mathrm{Sp}_{2 n}\right)$,

$$
C_{w} \subset \overline{C_{w^{\prime}}} \Longleftrightarrow w \leqslant w^{\prime} .
$$

The Schubert subvarieties of $\mathbb{F}_{\omega}(F)$ are the closures of the Schubert cells. The Schubert subvariety $X_{w}=\overline{C_{w}}$ is then given by

$$
X_{w}=\bigcup_{w^{\prime} \leqslant w} C_{w^{\prime}} .
$$

The Bruhat order on $W\left(\mathrm{Sp}_{2 n}\right)$ and the order on the sets of admissible indices $\mathbf{I}_{k, 2 n}^{\omega}$ are related by Proctor's criterion (cf. Pro82]) which states that, for $w, w^{\prime} \in$ $W\left(\operatorname{Sp}_{2 n}\right), w \leqslant w^{\prime}$ if and only if

$$
\{w(1), \ldots, w(i)\} \uparrow \leqslant\left\{w^{\prime}(1), \ldots, w^{\prime}(i)\right\} \uparrow
$$

for all $1 \leqslant i \leqslant n$. Geometrically this means that, for any $V_{\bullet}=\left(V_{1} \subset \cdots \subset V_{n}\right)$ in $\mathbb{F}_{\omega}(F), V_{\bullet} \in X_{w}$ if and only if $V_{i} \in X_{\{w(1), \ldots, w(i)\} \uparrow}$ for all $1 \leqslant i \leqslant n$. In terms of the incidence with the flag $E_{\bullet}$, the Schubert subvariety $X_{w}$ is the set of flags of isotropic subspaces $V_{\bullet}=\left(V_{1} \subset \cdots \subset V_{n}\right)$ which verify

$$
\operatorname{dim}\left(V_{i} \cap E_{j}\right) \geqslant r_{w}(i, j)
$$

for all $1 \leqslant i \leqslant n$ and all $1 \leqslant j \leqslant 2 n$.

\section{The ODD SYMPLECTIC GROUP}

Let $E$ be a complex vector space of dimension $2 n+1$ and $\omega \in \Lambda^{2} E^{*}$ a generic skew-form on $E$. More precisely, we assume that $\omega$ is of maximal rank, ie rank $\omega=$ $2 n$, since the skew-forms of maximal rank form an open orbit under the action of the general linear group $\mathrm{GL}(E)$ on $\Lambda^{2} E^{*}$.

Definition 3.1. The odd symplectic group $\mathrm{Sp}_{2 n+1}$ is the stabilizer in $\mathrm{GL}(E)$ of the skew-form $\omega$. 
Being an isotropy group for an algebraic action, $\mathrm{Sp}_{2 n+1}$ is a closed subgroup of $\mathrm{GL}(E)$. Let $R$ be the one dimensional kernel of $\omega$ with a generator $e_{0}$, choose a supplement $F$, so that the restriction of $\omega$ to $F$ is non degenerate, and take $\left\{e_{1}, \ldots, e_{2 n}\right\}$ to be a symplectic basis of $F$. Then in the basis $\left\{e_{0}, \ldots, e_{2 n}\right\}$ the skew-form $\omega$ is given by

$$
\omega\left(e_{i}, e_{j}\right)=\delta_{i, 2 n+1-j}, \quad \text { for all } 0 \leqslant i, j \leqslant 2 n .
$$

We will call such a basis an odd symplectic basis. We continue to use the notation $\bar{\imath}=2 n+1-i$ except that now we consider $\overline{0}$ to be not defined.

With respect to the decomposition $R \oplus F=E$ the odd symplectic group is the group of the matrices of the form

$$
\left(\begin{array}{ll}
\lambda & \ell \\
0 & a
\end{array}\right)
$$

where $\lambda \in \mathbb{C}^{*}, a \in \mathrm{Sp}_{2 n}, \ell \in \mathbb{C}^{2 n}$. From now on, we fix the odd symplectic basis $\left\{e_{0}, e_{1}, \ldots, e_{2 n}\right\}$ and identify $\mathrm{Sp}_{2 n+1}$ with this group of matrices.

The symplectic group $\mathrm{Sp}_{2 n}$ embeds in $\mathrm{Sp}_{2 n+1}$ as those matrices (2) with $\lambda=1$ and $\ell=0$. Denote $U$ the subgroup of $\operatorname{Sp}_{2 n+1}$ given by the matrices (2) with $\lambda=1$ and $a=1$. It is isomorphic to the additive group $\left(\mathbb{C}^{2 n},+\right)$ and we easily check that it is a normal subgroup in $\mathrm{Sp}_{2 n+1}$. Therefore the odd symplectic group $\mathrm{Sp}_{2 n+1}$ is equal to the semi-direct product

$$
\left(\mathbb{C}^{*} \times \mathrm{Sp}_{2 n}\right) \ltimes U
$$

of his two subgroups $\mathbb{C}^{*} \times \mathrm{Sp}_{2 n}$ and $U$. It follows that $\mathrm{Sp}_{2 n+1}$ is connected and of dimension $(n+1)(2 n+1)$. With the action of $\mathbb{C}^{*} \times \mathrm{Sp}_{2 n}$ by interior automorphisms, $U$ is isomorphic to the dual $F^{*}$ of the standard representation of $\operatorname{Sp}_{2 n}$ (where $\mathbb{C}^{*}$ acts by homotheties).

Since $U$ is a normal unipotent subgroup, it follows that $\mathrm{Sp}_{2 n+1}$ is not reductive. Actually $U$ is the unipotent radical $R_{u}$ of $\mathrm{Sp}_{2 n+1}$, since on the one hand we have $U \subset R_{u}$ and on the other hand the quotient $\mathrm{Sp}_{2 n+1} / U \simeq \mathbb{C}^{*} \times \mathrm{Sp}_{2 n}$ is reductive so the image of $R_{u}$ in this quotient is trivial.

We describe now the Borel subgroups and the maximal tori of $\mathrm{Sp}_{2 n+1}$.

Proposition 3.2. Let $B$ be the subgroup of $\mathrm{Sp}_{2 n+1}$ of upper triangular matrices in the odd symplectic basis $\left\{e_{0}, e_{1}, \ldots, e_{2 n}\right\}$. Then $B$ is a Borel subgroup.

Proof. $B$ is solvable since it is a subgroup of the subgroup of upper triangular matrices in $\operatorname{GL}(2 n+1, \mathbb{C})$. It is given by the matrices of the form

$$
\left(\begin{array}{ll}
\lambda & \ell \\
0 & a
\end{array}\right)
$$

with $a \in \operatorname{Sp}_{2 n}$ upper triangular, so $B$ is the semi-direct product $B=\left(\mathbb{C}^{*} \times B_{2 n}\right) \ltimes U$ where $B_{2 n} \subset \mathrm{Sp}_{2 n}$ is the Borel subgroup of $\mathrm{Sp}_{2 n}$ preserving the flag generated by the symplectic basis $\left\{e_{1}, \ldots, e_{2 n}\right\}$. In particular $B$ is connected and therefore it is contained in a Borel subgroup $\tilde{B}$. Since $\tilde{B}$ contains $U$, it is the semi-direct product $\tilde{B}=\left[\tilde{B} \cap\left(\mathbb{C}^{*} \times \mathrm{Sp}_{2 n}\right)\right] \ltimes U$. But $\tilde{B} \cap\left(\mathbb{C}^{*} \times \mathrm{Sp}_{2 n}\right)$ is connected (since $\tilde{B}$ and $U$ are) and solvable and contains $\mathbb{C}^{*} \times B_{2 n}$. It follows that $\tilde{B} \cap\left(\mathbb{C}^{*} \times \mathrm{Sp}_{2 n}\right)=\mathbb{C}^{*} \times B_{2 n}$, and this means that $\tilde{B}=B$.

Proposition 3.3. Let $T$ be the subgroup of $\mathrm{Sp}_{2 n+1}$ of diagonal matrices in the odd symplectic basis $\left\{e_{0}, e_{1}, \ldots, e_{2 n}\right\}$. Then $T$ is a maximal torus.

Proof. $T$ is a torus since it is a group of diagonal matrices. We have $T=\mathbb{C}^{*} \times$ $T_{2 n}$ where $T_{2 n} \subset \mathrm{Sp}_{2 n}$ is the maximal torus of $\mathrm{Sp}_{2 n}$ of diagonal matrices in the 
symplectic basis $\left\{e_{1}, \ldots, e_{2 n}\right\}$. It suffices to show that $T$ is equal to its centralizer. An element

$$
\left(\begin{array}{ll}
\lambda & \ell \\
0 & a
\end{array}\right)
$$

of $\mathrm{Sp}_{2 n+1}$ centralizes $T$ if and only if $a d=d a$ and $\ell d=t \ell$ for all $(t, d) \in \mathbb{C}^{*} \times T_{2 n}$, ie $a \in C_{\mathrm{Sp}_{2 n}}\left(T_{2 n}\right)$ and $\ell=0$. Since $C_{\mathrm{Sp}_{2 n}}\left(T_{2 n}\right)=T_{2 n}$, we are done.

Note that the elements of the maximal torus $T$ are the diagonal matrices

$$
\operatorname{diag}\left(t_{0}, t_{1}, \ldots, t_{n}, t_{n}^{-1}, \ldots, t_{1}^{-1}\right), \quad t_{0}, \ldots, t_{n} \in \mathbb{C}^{*} .
$$

Remark 3.4. The last proof shows that $C_{\mathrm{Sp}_{2 n+1}}(T)=T$ so the Weyl group of $\mathrm{Sp}_{2 n+1}$ is the quotient $N_{\mathrm{Sp}_{2 n+1}}(T) / T$. This is actually isomorphic to the Weyl group of the symplectic group $\mathrm{Sp}_{2 n}$. This follows from the fact that $N_{\mathrm{Sp}_{2 n+1}}(T)=$ $\mathbb{C}^{*} \times N_{\mathrm{Sp}_{2 n}}\left(T_{2 n}\right)$, which we can easily check.

For later use, we record the following :

Proposition 3.5. The center of $\mathrm{Sp}_{2 n+1}$ is $\{ \pm \mathrm{Id}\}$.

3.6. Relating $\mathrm{Sp}_{2 n+1}$ and $\mathrm{Sp}_{2 n+2}$. Embed $E$ as a hyperplane in a complex vector space $\tilde{E}$ of dimension $2 n+2$. Then the odd symplectic form $\omega$ extends to a symplectic form $\tilde{\omega}$ on $\tilde{E}$. Indeed it suffices to take a vector $e_{\overline{0}}$ in $\tilde{E}$ not belonging to $E$ and to define $\tilde{\omega}$ in such a way that the basis $\left\{e_{0}, e_{1}, \ldots, e_{n}, e_{\bar{n}}, \ldots, e_{\overline{1}}, e_{\overline{0}}\right\}$ be symplectic. Denote simply by $\mathrm{Sp}_{2 n+2}$ the symplectic group which fixes $\tilde{\omega}$. Let $P$ be the parabolic subgroup of $\mathrm{Sp}_{2 n+2}$ which preserves the line $R$. Then $P$ also preserves $E$ which is the orthogonal of $R$, and for any element $g \in P$ the restriction $\left.g\right|_{E}$ preserves $\omega$, ie is in $\mathrm{Sp}_{2 n+1}$.

Proposition 3.7. The morphism $P \rightarrow \mathrm{Sp}_{2 n+1}$ given by $\left.g \mapsto g\right|_{E}$ is surjective.

Proof. In the symplectic basis $\left\{e_{0}, e_{1}, \ldots, e_{n}, e_{\bar{n}}, \ldots, e_{\overline{1}}, e_{\overline{0}}\right\}$ an element of $P$ is a matrix of the form

$$
\left(\begin{array}{lll}
\lambda & \ell & \nu \\
0 & a & c \\
0 & 0 & \mu
\end{array}\right)
$$

with $a \in \mathrm{Sp}_{2 n}, \lambda, \mu \in \mathbb{C}^{*}, \nu \in \mathbb{C}$ and $\ell, c \in \mathbb{C}^{2 n}$ a row and, respectively, column vector. The condition that this be an element of $\mathrm{Sp}_{2 n+1}$ is that the columns make a symplectic basis. Since $\left.g\right|_{E} \in \mathrm{Sp}_{2 n+1}$, we only need to look at the conditions involving the last column and this gives $\lambda \mu=1$ and, in matrix form,

$$
\mu \ell+\left(c_{2 n}, \ldots, c_{n+1},-c_{n}, \ldots,-c_{1}\right) a=0 .
$$

This shows that given any $(\lambda, \ell, a)$ there is a $c$ such that (3) belongs to $\operatorname{Sp}_{2 n+2}$ and we are done.

Remark 3.8. In the proof we see that giving $(\lambda, \ell, a)$ uniquely determines $c$ while $\nu$ is arbitrary. We can show that there is no way of choosing a $\nu$ for each $(\lambda, \ell, a)$ such that the resulting injection $\mathrm{Sp}_{2 n+1} \rightarrow P$ be a morphism of algebraic groups, ie the morphism $P \rightarrow \mathrm{Sp}_{2 n+1}$ has no section. Actually we can show that $\mathrm{Sp}_{2 n+1}$ cannot be embedded as an algebraic subgroup in $\mathrm{Sp}_{2 n+2}$. For a proof of these statements, see [Mih05 ch. 1].

Remark 3.9. Through the morphism $P \rightarrow \mathrm{Sp}_{2 n+1}$, the Borel subgroup of $\mathrm{Sp}_{2 n+2}$ of upper triangular matrices in the symplectic basis $\left\{e_{0}, e_{1}, \ldots, e_{n}, e_{\bar{n}}, \ldots, e_{\overline{1}}, e_{\overline{0}}\right\}$ surjects onto the Borel subgroup of $\mathrm{Sp}_{2 n+1}$ of upper triangular matrices in the odd symplectic basis $\left\{e_{0}, e_{1}, \ldots, e_{n}, e_{\bar{n}}, \ldots, e_{\overline{1}}\right\}$. Similarly, the morphism $P \rightarrow \mathrm{Sp}_{2 n+1}$ 
restricts to an isomorphism between the maximal torus of $\mathrm{Sp}_{2 n+2}$ of diagonal matrices in the basis $\left\{e_{0}, e_{1}, \ldots, e_{n}, e_{\bar{n}}, \ldots, e_{\overline{1}}, e_{\overline{0}}\right\}$ and the maximal torus of $\mathrm{Sp}_{2 n+1}$ of diagonal matrices in the basis $\left\{e_{0}, e_{1}, \ldots, e_{n}, e_{\bar{n}}, \ldots, e_{\overline{1}}\right\}$.

Remark 3.10. From (4) it follows that the kernel of the morphism $P \rightarrow \mathrm{Sp}_{2 n+1}$ is the unipotent group of dimension one whose elements are the matrices of the form

$$
\left(\begin{array}{lll}
1 & 0 & \nu \\
0 & 1 & 0 \\
0 & 0 & 1
\end{array}\right)
$$

with $\nu \in \mathbb{C}$.

3.11. The intermediate odd symplectic group. Since $\mathrm{Sp}_{2 n+1}$ does not embed in $\mathrm{Sp}_{2 n+2}$ as an algebraic subgroup, it is desirable to have an alternative odd symplectic group which sits between $\mathrm{Sp}_{2 n}$ and $\mathrm{Sp}_{2 n+2}$. Such a group has been introduced by Gelfand and Zelevinski in GZ84. They notice that for any complex vector space $V$, the general linear group $\mathrm{GL}(V)$ has an open orbit in the space $V^{*} \oplus \Lambda^{2} V^{*}$, which is

$$
\{(\ell, \theta) \mid \ell \neq 0, \theta \text { of maximal rank and } \operatorname{ker} \ell \cap \operatorname{ker} \theta=(0)\},
$$

and they define the group $G(V)$ to be the isotropy group of this orbit. It is easy to check that if $V$ is of dimension $2 n+1$ then $G(V)$ is isomorphic to $\operatorname{Sp}_{2 n}$, while if $V$ is of dimension $2 n+2$ then $G(V)$ is isomorphic to the subgroup of $\mathrm{Sp}_{2 n+2}$ which fixes a non-zero element of $V^{*}$ (or, equivalently, of $V$ ). In the latter case, we will use the following designation for $G(V)$ :

Definition 3.12. The intermediate odd symplectic group $\widetilde{\mathrm{Sp}}_{2 n+1}$ is the stabilizer in $\mathrm{Sp}_{2 n+2}$ of a non-zero element of $\mathbb{C}^{2 n+2}$.

Since $\widetilde{\mathrm{Sp}}_{2 n+1}$ is well defined up to a conjugation, we can assume that the element it fixes is the first vector $e_{0}$ of the symplectic basis $\left\{e_{0}, e_{1}, \ldots, e_{n}, e_{\bar{n}}, \ldots, e_{\overline{1}}, e_{\overline{0}}\right\}$ so that $\widetilde{\mathrm{Sp}}_{2 n+1}$ identifies with the subgroup of the parabolic $P$ considered above given by the matrices (3) with $\lambda=\mu=1$. Its image via the morphism $P \rightarrow \operatorname{Sp}_{2 n+1}$ is the subgroup $\mathrm{SSp}_{2 n+1}$ of elements of determinant 1 in $\mathrm{Sp}_{2 n+1}$.

\section{ODD SYMPLECTIC FLAG MANIFOLDS}

We introduce here our main objects of study. For a sequence of integers $1 \leqslant$ $d_{1}<\cdots<d_{r} \leqslant n+1$, denote by $\mathbb{F}_{\omega}\left(d_{1}, \ldots, d_{r}, E\right)$ the variety of flags

$$
\left\{\left(V_{d_{1}} \subset \cdots \subset V_{d_{r}} \subset E\right) \mid \operatorname{dim} V_{d_{i}}=d_{i}, V_{d_{i}} \text { isotropic for all } 1 \leqslant i \leqslant r\right\} .
$$

These are the odd symplectic flag manifolds.

Let us first note that the condition that the integers $d_{i}$ be at most $n+1$ comes from the fact that $n+1$ is the maximal dimension of an isotropic subspace of $E$. Actually, a maximal isotropic subspace of $E$ is always of dimension $n+1$ since it contains the kernel $R$ and its image in $E / R$ is a maximal isotropic subspace.

When $r=1$ we will write $G_{\omega}(k, E)$ instead of $\mathbb{F}_{\omega}(k, E)$ and call these varieties odd symplectic grassmannians. These are simply the grassmannians of isotropic subspaces of $E$. When $r=n+1$ we denote $\mathbb{F}_{\omega}(E)=\mathbb{F}_{\omega}(1, \ldots, n+1, E)$ and call this, if there is no risk of confusion, the odd symplectic flag manifold. This is the variety of maximal flags of isotropic subspaces of $E$.

Since the isomorphism class of the variety $\mathbb{F}_{\omega}\left(d_{1}, \ldots, d_{r}, E\right)$ depends only on the dimension of $E$, we will also denote this variety by $\mathbb{F}_{\omega}\left(d_{1}, \ldots, d_{r} ; 2 n+1\right)$ (and correspondingly, we will also use the notations $G_{\omega}(k, 2 n+1)$ and $\left.\mathbb{F}_{\omega}(2 n+1)\right)$.

The variety $\mathbb{F}_{\omega}\left(d_{1}, \ldots, d_{r}, E\right)$ is a closed subvariety of $\mathbb{F}\left(d_{1}, \ldots, d_{r}, E\right)$, the type A flag manifold defined by forgetting the isotropy condition, therefore the odd 
symplectic flag manifolds are projective varieties. The odd symplectic group acts naturally on the varieties $\mathbb{F}_{\omega}\left(d_{1}, \ldots, d_{r}, E\right)$, the action being defined by restricting to $\mathbb{F}_{\omega}\left(d_{1}, \ldots, d_{r}, E\right)$ the usual action of $\mathrm{GL}(E)$ on the flag manifold $\mathbb{F}\left(d_{1}, \ldots, d_{r}, E\right)$. The major difference between this situation and the one we have in the symplectic setting is that this action is not transitive. The reason, as explained in the introduction, is that the kernel $R$ is fixed by $\mathrm{Sp}_{2 n+1}$ and so flags which do not have the same incidence with $R$ cannot be conjugated by $\operatorname{Sp}_{2 n+1}$ (we still have to prove that these different incidence types actually occur, but this will become clear below). We will show that actually these incidence conditions suffice to describe the orbits.

Being non homogeneous, it is no longer granted that the odd symplectic flag varieties $\mathbb{F}_{\omega}\left(d_{1}, \ldots, d_{r}, E\right)$ are smooth. It turns out that they actually are:

Proposition 4.1. The odd symplectic flag manifold $\mathbb{F}_{\omega}\left(d_{1}, \ldots, d_{r}, E\right)$ is a smooth subvariety of codimension $\frac{1}{2} d_{r}\left(d_{r}-1\right)$ in the flag manifold $\mathbb{F}\left(d_{1}, \ldots, d_{r}, E\right)$.

Proof. Let $T=T_{d_{r}}$ denote the highest rank tautological bundle on the flag manifold $\mathbb{F}\left(d_{1}, \ldots, d_{r}, E\right)$. The fiber of $T$ at a point $V_{\bullet}=\left(V_{d_{1}} \subset \cdots \subset V_{d_{r}}\right)$ is $V_{d_{r}}$. Any skew-form $\theta \in \Lambda^{2} E^{*}$ can be seen as a global section of $\Lambda^{2} T^{*}$, whose value at a point $V_{\bullet}=\left(V_{d_{1}} \subset \cdots \subset V_{d_{r}}\right)$ is the restriction $\left.\theta\right|_{V_{d_{r}}} \in \Lambda^{2} V_{d_{r}}^{*}$. In this way the section defined by $\theta \in \Lambda^{2} E^{*}$ vanishes at a point $V_{\bullet}$ if and only if $V_{d_{r}}$ is isotropic with respect to $\theta$. Therefore the zero locus of our odd symplectic form $\omega$ seen as a section of $\Lambda^{2} T^{*}$ is $\mathbb{F}_{\omega}\left(d_{1}, \ldots, d_{r}, E\right)$. Now, the vector bundle $\Lambda^{2} T^{*}$ is generated by its global sections which come from $\Lambda^{2} E^{*}$ since any skew-form defined on a subspace $V_{d_{r}}$ extends to $E$. The section defined by the odd symplectic form $\omega$ is generic among the sections which come from $\Lambda^{2} E^{*}$, so to conclude by Bertini's theorem it suffices to show that $\omega$ vanishes in at least a point of $\mathbb{F}\left(d_{1}, \ldots, d_{r}, E\right)$. For this it is enough to take the point $\left(E_{d_{1}} \subset \cdots \subset E_{d_{r}}\right)$ where $E_{\bullet}$ is the complete flag generated by the odd symplectic basis $\left\{e_{0}, e_{1}, \ldots, e_{2 n}\right\}$.

4.2. $\mathrm{Sp}_{2 n+1}$-orbits in the odd symplectic grassmannians. Any isotropic subspace $V \subset E$ of dimension $n+1$ contains the kernel $R$ and is of the form $V=R \oplus W$ with $W \subset F$ isotropic of dimension $n$, so the odd symplectic grassmannian $G_{\omega}(n+1, E)$ is isomorphic to the symplectic grassmannian $G_{\omega}(n, F)$. Therefore $G_{\omega}(n+1, E)$ is already homogeneous under the subgroup $\operatorname{Sp}_{2 n} \subset \operatorname{Sp}_{2 n+1}$. For the other odd symplectic grassmannians, the $\mathrm{Sp}_{2 n+1}$-orbits are given by the incidence with the kernel $R=\mathbb{C} e_{0}$, that is we have:

Proposition 4.3. For $1 \leqslant k \leqslant n$ the odd symplectic group $\mathrm{Sp}_{2 n+1}$ acts on the odd symplectic grassmannian $G_{\omega}(k, 2 n+1)$ with two orbits

$$
\begin{aligned}
& X_{0}=\left\{V \in G_{\omega}(k, 2 n+1) \mid e_{0} \in V\right\} \\
& X_{1}=\left\{V \in G_{\omega}(k, 2 n+1) \mid e_{0} \notin V\right\} .
\end{aligned}
$$

The closed orbit $X_{0}$ is isomorphic to the symplectic grassmannian $G_{\omega}(k-1,2 n)$ and the open orbit $X_{1}$ is isomorphic to the total space of the dual of the tautological bundle on the symplectic grassmannian $G_{\omega}(k, 2 n)$.

Proof. Both $X_{0}$ and $X_{1}$ are obviously stable under $\operatorname{Sp}_{2 n+1}$. Any $V \in X_{0}$ is of the form $V=R \oplus W$ with $W=V \cap F$ isotropic of dimension $k-1$, so $X_{0}$ is isomorphic to the symplectic grassmannian $G_{\omega}(k-1, F)$ and is already an orbit of the subgroup $\mathrm{Sp}_{2 n} \subset \mathrm{Sp}_{2 n+1}$.

Let now $p: E \rightarrow F$ be the projection coming from the decomposition $E=R \oplus F$. For any $V \in X_{1}, p(V) \subset F$ is an isotropic subspace of dimension $k$. We get in this way a map

$$
p: X_{1} \longrightarrow G_{\omega}(k, F)
$$


onto the symplectic grassmannian $G_{\omega}(k, F)$ which we regard as a subvariety in $G_{\omega}(k, E)$. If $V=\left\langle v_{1}, \ldots, v_{k}\right\rangle \in X_{1}$, with $v_{i}=\alpha_{i} e_{0}+v_{i}^{\prime}, v_{i}^{\prime} \in F$, then $p(V)=$ $\left\langle v_{1}^{\prime}, \ldots, v_{k}^{\prime}\right\rangle$. Therefore $V$ and $p(V)$ will be conjugated by any element of the unipotent radical $U$ of $\mathrm{Sp}_{2 n+1}$ which sends $v_{i}^{\prime} \mapsto v_{i}^{\prime}+\alpha_{i} e_{0}$ for all $1 \leqslant i \leqslant k$. Such an element exist since the $v_{i}^{\prime}$ are independent. Conversely, for any $g \in U$ we have $p(g . V)=p(V)$, and so the orbits of $U$ in $X_{1}$ coincide with the fibers of $p: X_{1} \rightarrow G_{\omega}(k, F)$. In particular, any orbit of $U$ in $X_{1}$ meets $G_{\omega}(k, F) \subset X_{1}$ which is an orbit of $\operatorname{Sp}_{2 n} \subset \mathrm{Sp}_{2 n+1}$, and therefore $\mathrm{Sp}_{2 n} \ltimes U \subset \mathrm{Sp}_{2 n+1}$ acts transitively in $X_{1}$.

Let now $T$ be the tautological bundle on the symplectic grassmannian $G_{\omega}(k, F)$. The fiber of the dual $T^{*}$ at a point $W \in G_{\omega}(k, F)$ is $W^{*}$. We define a map $T^{*} \rightarrow X_{1}$ by sending an element $\ell \in W^{*}$ to its graph $\Gamma_{\ell} \subset W \oplus \mathbb{C}=W \oplus R \subset F \oplus R=E$ which is an isotropic subspace of $E$ which does not contain $e_{0}$ and for which $p\left(\Gamma_{\ell}\right)=W$. In coordinates, if $W=\left\langle w_{1}, \ldots, w_{k}\right\rangle$ then $\Gamma_{\ell}=\left\langle w_{1}+\ell\left(w_{1}\right) e_{0}, \ldots, w_{k}+\ell\left(w_{k}\right) e_{0}\right\rangle$. Conversely, if $V \in X_{1}$ then $V \subset p(V) \oplus \mathbb{C} e_{0}$ is the graph of a linear map $p(V) \rightarrow$ $\mathbb{C} e_{0}=\mathbb{C}$, ie an element of the fiber of $T^{*}$ at the point $p(V)$.

4.4. $\mathrm{Sp}_{2 n+1}$-orbits in the odd symplectic flag manifold $\mathbb{F}_{\omega}(2 n+1)$. As for the odd symplectic grassmannians, the $\mathrm{Sp}_{2 n+1}$-orbits of the odd symplectic flag manifold $\mathbb{F}_{\omega}(E)$ are described by the incidence with the kernel $R=\mathbb{C} e_{0}$. More precisely, we have:

Proposition 4.5. The odd symplectic group $\mathrm{Sp}_{2 n+1}$ acts on the odd symplectic flag manifold $\mathbb{F}_{\omega}(2 n+1)$ with $n+1$ orbits

$$
\mathcal{O}_{i}=\left\{\left(V_{1} \subset \cdots \subset V_{n+1}\right) \in \mathbb{F}_{\omega}(2 n+1) \mid e_{0} \in V_{i}, e_{0} \notin V_{i-1}\right\}, \quad 1 \leqslant i \leqslant n+1 .
$$

Moreover, for all $1 \leqslant i \leqslant n+1$, the orbit $\mathcal{O}_{i}$ is isomorphic to the total space of the dual $T_{i-1}^{*}$ of the rank $i-1$ tautological bundle on the symplectic flag manifold $\mathbb{F}_{\omega}(2 n)$.

Proof. The $\mathcal{O}_{i}$ are clearly $\mathrm{Sp}_{2 n+1}$-stable. Let again $p: E \rightarrow F$ be the projection coming from the decomposition $E=R \oplus F$. For any $V_{\bullet}=\left(V_{1} \subset \cdots \subset V_{n+1}\right)$ in $\mathcal{O}_{i}$, the projection $p$ restricts to isomorphisms $V_{j} \rightarrow p\left(V_{j}\right)$ for all $1 \leqslant j \leqslant i-1$, and we have $V_{j}=p\left(V_{j}\right) \oplus R$ with $p\left(V_{j}\right)=V_{j} \cap F$ for $i \leqslant j \leqslant n+1$. We also have $V_{i}=V_{i-1} \oplus R$ and $p\left(V_{i-1}\right)=p\left(V_{i}\right)$. Let's denote by $p_{i}\left(V_{\bullet}\right)$ the flag

$$
p_{i}\left(V_{\bullet}\right)=\left(p\left(V_{1}\right) \subset \cdots \subset p\left(V_{i-1}\right) \subset V_{i} \subset \cdots \subset V_{n+1}\right) .
$$

We get in this way a map $p_{i}$ from $\mathcal{O}_{i}$ onto the closed subvariety $Y_{i} \subset \mathcal{O}_{i}$

$$
Y_{i}=\left\{V_{\bullet}=\left(V_{1} \subset \cdots \subset V_{n+1}\right) \in \mathbb{F}_{\omega}(E) \mid V_{1}, \ldots, V_{i-1} \subset F, e_{0} \in V_{i}\right\} .
$$

The subvariety $Y_{i}$ is isomorphic to the symplectic flag manifold $\mathbb{F}_{\omega}(F)$ via the map which sends a flag $\left(W_{1} \subset \cdots \subset W_{n}\right) \in \mathbb{F}_{\omega}(F)$ to the flag

$$
\left(W_{1} \subset \cdots \subset W_{i-1} \subset W_{i-1} \oplus R \subset \cdots \subset W_{n} \oplus R\right) \in Y_{i} .
$$

In coordinates, if $\left\{v_{1}^{\prime}, \ldots, v_{n}^{\prime}\right\}$ is an adapted basis for the flag in $\mathbb{F}_{\omega}(F)$ which corresponds via the isomorphism above to the flag $p_{i}\left(V_{\bullet}\right)$, then

$$
\left\{v_{1}^{\prime}, \ldots, v_{i-1}^{\prime}, e_{0}, v_{i}^{\prime}, \ldots, v_{n}^{\prime}\right\}
$$

is an adapted basis for $p_{i}\left(V_{\bullet}\right)$, and there are $a_{1}, \ldots, a_{i-1} \in \mathbb{C}$ such that

$$
\left\{v_{1}^{\prime}+a_{1} e_{0}, \ldots, v_{i-1}^{\prime}+a_{i-1} e_{0}, e_{0}, v_{i}^{\prime}, \ldots, v_{n}^{\prime}\right\}
$$

is an adapted basis for the flag $V_{\bullet}$. It follows that $V_{\bullet}$ and $p_{i}\left(V_{\bullet}\right)$ will be conjugated by any element of the unipotent radical $U$ of $\operatorname{Sp}_{2 n+1}$ which sends $v_{j}^{\prime} \mapsto v_{j}^{\prime}+a_{j} e_{0}$ for all $1 \leqslant j \leqslant i-1$. Such an element exists since $v_{1}^{\prime}, \ldots, v_{i-1}^{\prime}$ are independent. Conversely, for any $g \in U$ we have $p_{i}\left(V_{\bullet}\right)=p_{i}\left(g \cdot V_{\bullet}\right)$, so the orbits of $U$ in $\mathcal{O}_{i}$ coincide with the fibers of $p_{i}$. Therefore any orbit of $U$ in $\mathcal{O}_{i}$ meets $Y_{i} \subset \mathcal{O}_{i}$ 
which is an orbit of $\mathrm{Sp}_{2 n} \subset \mathrm{Sp}_{2 n+1}$, and so it follows that $\mathrm{Sp}_{2 n} \ltimes U \subset \mathrm{Sp}_{2 n+1}$ acts transitively on $\mathcal{O}_{i}$.

Let now $T_{i-1}$ be the tautological bundle of rank $i-1$ on the symplectic flag manifold $\mathbb{F}_{\omega}(F)$. The fiber of the dual $T_{i-1}^{*}$ at a point $W_{\bullet}=\left(W_{1} \subset \cdots \subset W_{n}\right) \in$ $\mathbb{F}_{\omega}(F)$ is $W_{i-1}^{*}$. We define a map $T_{i-1}^{*} \rightarrow \mathcal{O}_{i}$ by sending an element $\ell \in W_{i-1}^{*}$ to the flag $V_{\bullet}=\left(V_{1} \subset \cdots \subset V_{n+1}\right) \in \mathcal{O}_{i}$ where: $V_{i-1}$ is the graph of $\ell, \Gamma_{\ell} \subset$ $W_{i-1} \oplus \mathbb{C}=W_{i-1} \oplus R \subset F \oplus R=E$; for $1 \leqslant j \leqslant i-2, V_{j}$ is the graph $\Gamma_{\ell_{W_{W_{j}}}} \subset \Gamma_{\ell}$ of the restriction $\left.\ell\right|_{W_{j}}$; and $V_{j}=W_{j-1} \oplus R$ for $i \leqslant j \leqslant n+1$. In coordinates, if $\left\{v_{1}^{\prime}, \ldots, v_{n}^{\prime}\right\}$ is an adapted basis for the flag $W \bullet$ then

$$
\left\{v_{1}^{\prime}+\ell\left(v_{1}^{\prime}\right) e_{0}, \ldots, v_{i-1}^{\prime}+\ell\left(v_{i-1}^{\prime}\right) e_{0}, e_{0}, v_{i}^{\prime}, \ldots, v_{n}^{\prime}\right\}
$$

is an adapted basis for $V_{\bullet}$. Conversely, for $V_{\bullet}=\left(V_{1} \subset \cdots \subset V_{n+1}\right) \in \mathcal{O}_{i}$ let $W_{\bullet}=\left(W_{1} \subset \cdots \subset W_{n}\right)$ be the flag in $\mathbb{F}_{\omega}(F)$ corresponding via the isomorphism $\mathbb{F}_{\omega}(F) \simeq Y_{i}$ to the flag $p_{i}\left(V_{\bullet}\right)$, ie

$$
W_{\bullet}=\left(p\left(V_{1}\right) \subset \cdots \subset p\left(V_{i-1}\right) \subset p\left(V_{i+1}\right) \subset \cdots \subset p\left(V_{n+1}\right)\right) .
$$

Then $V_{i-1}$ is the graph of a linear map $\ell: W_{i-1} \rightarrow \mathbb{C} e_{0}=\mathbb{C}$, ie an element of the fiber of $T_{i-1}^{*}$ at the point $W_{\bullet}$, and we define the inverse map $\mathcal{O}_{i} \rightarrow T_{i-1}^{*}$ by sending $V$. to $\ell$.

Proposition 4.6. The closures of the $\mathrm{Sp}_{2 n+1}$-orbits of $\mathbb{F}_{\omega}(2 n+1)$ are the subvarieties

$$
\overline{\mathcal{O}_{i}}=\left\{\left(V_{1} \subset \cdots \subset V_{n+1}\right) \in \mathbb{F}_{\omega}(2 n+1) \mid e_{0} \in V_{i}\right\}, \quad 1 \leqslant i \leqslant n+1
$$

and are smooth.

Proof. The first assertion is clear. Now let $\pi_{i}: \mathbb{F}_{\omega}(E) \rightarrow G_{\omega}(i, E)$ be the map $\left(V_{1} \subset \cdots \subset V_{n+1}\right) \mapsto V_{i}$. The image $\pi_{i}\left(\overline{\mathcal{O}_{i}}\right)$ is the closed $\operatorname{Sp}_{2 n+1}$-orbit $X_{0}$ in the odd symplectic grassmannian $G_{\omega}(i, E)$. For $V_{i} \in X_{0}$, the fiber $\pi_{i}^{-1}\left(V_{i}\right)$ is isomorphic to $\mathbb{F}\left(V_{i}\right) \times \mathbb{F}_{\bar{\omega}}\left(V_{i}^{\perp} / V_{i}\right)$ where $\bar{\omega}$ is the form induced by $\omega$ on the quotient $V_{i}^{\perp} / V_{i}$. For any $V_{i} \in X_{0}$ we have $\operatorname{dim}\left(V_{i}^{\perp}\right)=2 n+2-i$ so $\pi_{i}: \mathcal{O}_{i} \rightarrow X_{0}$ is a fibration with fiber $\mathbb{F}(i) \times \mathbb{F}_{\omega}(2(n+1-i))$ over the smooth base $X_{0} \simeq G_{\omega}(i-1,2 n)$.

Note that from the second assertion of the proposition 4.5] it follows that $\mathcal{O}_{i}$ is of codimension $n+1-i$ in $\mathbb{F}_{\omega}(2 n+1)$. In particular, $\overline{\mathcal{O}_{n}}=\mathbb{F}_{\omega}(2 n+1) \backslash \mathcal{O}_{n+1}$ is an irreducible $\mathrm{Sp}_{2 n+1}$-stable divisor.

Remark 4.7. The $\mathrm{Sp}_{2 n+1}$-orbits in the other ("partial") odd symplectic flag manifolds satisfy the obvious analogs of propositions 4.5 and 4.6

4.8. Schubert cells in odd symplectic grassmannians. Since the odd symplectic grassmannian $G_{\omega}(k, E)$ is a subvariety of the usual grassmannian $G(k, E)$, it seems natural to try to define "Schubert cells" in $G_{\omega}(k, E)$ by using incidence conditions with respect to some fixed flag, ie to take Schubert cells in $G(k, E)$ and intersect them with $G_{\omega}(k, E)$. And indeed, if the fixed flag is generated by an odd symplectic basis, we can readily show that the "Schubert cells" we obtain in this way can be described in terms of row echelon matrices, as for the symplectic grassmannians, and so prove, for example, that they are isomorphic to affine spaces. It is then a bit trickier, though, to derive the incidence relations between the cells, ie the Bruhat order on the set of cells. Things simplify noticeably once we observe that the odd symplectic grassmannian $G_{\omega}(k, E)$ can be identified with a Schubert subvariety of a symplectic grassmannian $G_{\omega}(k, 2 n+2)$.

Let indeed, as in 3.6 $\tilde{E}$ be a complex vector space of dimension $2 n+2$ which contains $E$ as a hyperplane and $\tilde{\omega}$ a symplectic form on $\tilde{E}$ which extends $\omega$. Then, 
clearly, an element $V \in G_{\omega}(k, E)$ is nothing but an isotropic subspace of $\tilde{E}$ which is contained in the hyperplane $E$, that is, $G_{\omega}(k, E)$ identifies with the subvariety

$$
\left\{V \in G_{\tilde{\omega}}(k, \tilde{E}) \mid V \subset E\right\} .
$$

This is a Schubert subvariety in $G_{\tilde{\omega}}(k, \tilde{E})$. Let $e_{2 n+1} \in \tilde{E}$ be such that the basis $\left\{e_{0}, \ldots, e_{2 n}, e_{2 n+1}\right\}$ is symplectic and let $E_{\text {e }}$ be the flag generated by this basis. Then $E=E_{2 n+1}$ and in the notation of 2.11] the subvariety (6) is the Schubert subvariety $X_{\bar{k} \ldots \overline{2} \overline{1}}$ if $k<n+1$, respectively $X_{0 \bar{n} \ldots \overline{2} \overline{1}}$ if $k=n+1$. We denote by $\max _{k, n}$ the multi-index of the Schubert variety (6) that is

$$
\max _{k, n}= \begin{cases}(\bar{k}, \ldots, \overline{1}) & \text { if } k<n+1 \\ (0, \bar{n}, \ldots, \overline{1}) & \text { if } k=n+1\end{cases}
$$

We will actually suppose that $k$ and $n$ are fixed and slightly abuse notation and write $\max$ instead of $\max _{k, n}$.

Through its identification with the Schubert variety $X_{\max }$, the odd symplectic grassmannian $G_{\omega}(k, E)$ acquires the cell decomposition of the latter

$$
X_{\max }=\bigcup_{I \leqslant \max } C_{I} .
$$

We then define the Schubert cells of $G_{\omega}(k, E)$ (with respect to the flag $\left(E_{1} \subset \cdots \subset\right.$ $\left.E_{2 n+1}\right)$ ) to be the Schubert cells of $G_{\tilde{\omega}}(k, \tilde{E})$ (with respect to the flag $\left(E_{1} \subset \cdots \subset\right.$ $\left.E_{2 n+1} \subset E_{2 n+2}\right)$ ) which are included in $X_{\text {max }}$.

We check easily that for $I \in \mathbf{I}_{k, 2 n+2}^{\omega}, I=\left(0 \leqslant i_{1}<\cdots<i_{k} \leqslant 2 n+1\right)$, we have $I \leqslant \max$ if and only if $i_{k} \leqslant 2 n$, that is if $I$ is a multi-index in the set $\{0, \ldots, 2 n\}$. So let us introduce the following notation:

Notation 4.9. Let $\mathbf{I}_{k, 2 n+1}$ be the set of multi-indices $I=\left(0 \leqslant i_{1}<\cdots<i_{k} \leqslant 2 n\right)$ and $\mathbf{I}_{k, 2 n+1}^{\omega}$ the subset of admissible indices, ie indices $I$ such that, for each $1 \leqslant i \leqslant$ $n$, at most one of $i$ or $\bar{\imath}$ appears in $I$.

Then $\mathbf{I}_{k, 2 n+1}^{\omega}$ identifies with the interval $\left\{I \in \mathbf{I}_{k, 2 n+2}^{\omega} \mid I \leqslant \mathbf{m a x}\right\}$ so the Schubert cells of $G_{\omega}(k, E)$ are parametrized by $\mathbf{I}_{k, 2 n+1}^{\omega}$. We state now the characterization of the Schubert cells of $G_{\omega}(k, E)$ by incidence conditions with respect to the flag $\left(E_{1} \subset \cdots \subset E_{2 n+1}\right)$ :

Proposition 4.10. The Schubert cell $C_{I}$ of $G_{\omega}(k, E)$ associated to an admissible multi-index $I \in \mathbf{I}_{k, 2 n+1}^{\omega}$ is the set of isotropic subspaces of dimension $k$ in $E$ whose incidence type is $I$, ie

$$
C_{I}=\left\{V \in G_{\omega}(k, 2 n+1) \mid \operatorname{dim}\left(V \cap E_{i}\right)=\alpha, \quad i_{\alpha} \leqslant i<i_{\alpha+1}, 1 \leqslant \alpha \leqslant k\right\},
$$

where we put $i_{k+1}=2 n+1$.

Proof. Indeed, these incidence conditions are obtained from those defining $C_{I}$ in $G_{\tilde{\omega}}(k, \tilde{E})$ by forgetting the last one (corresponding to $\left.i=2 n+1\right)$, which is superfluous since $i_{k} \leqslant 2 n$.

4.11. Similarly to the symplectic case, the Schubert cell $C_{I}$ in $G_{\omega}(k, E)$ is the set of isotropic subspaces of dimension $k$ in $E$ which, with respect to the odd symplectic basis $\left\{e_{0}, \ldots, e_{2 n}\right\}$, can be generated by the rows of a row-echelon matrix of type $I$, ie a $k \times(2 n+1)$ matrix $\left(a_{\alpha i}\right)$ for which each entry $a_{\alpha i_{\alpha}}$ is non zero and all entries to its right are zero. For each $V \in C_{I}$ there is a unique such matrix verifying further the conditions that the entries $a_{\alpha i_{\alpha}}$ be 1 and all the entries below be zero. The remaining entries are free, except the entries below an entry opposed to a 1 (ie below an entry of the form $\left.a_{\alpha \overline{i_{\alpha}}}\right)$ which are determined. The free entries provide explicitly an isomorphism between $C_{I}$ and the affine space $\mathbb{A}^{\operatorname{dim} C_{I}}$. 
For example, in the odd symplectic grassmannian $G_{\omega}(3,9)$ the Schubert cell defined by the multi-index $(4,6,8)=(4, \overline{3}, \overline{1})$ is given by the row-space of the matrix

$$
\left(\begin{array}{lllllllll}
* & * & * & * & 1 & 0 & 0 & 0 & 0 \\
* & * & * & * & 0 & \bullet & 1 & 0 & 0 \\
* & * & * & \bullet & 0 & \bullet & 0 & * & 1
\end{array}\right)
$$

We have marked by $*$ the free entries and by $\bullet$ those which are determined.

4.12. Let $B$ be the Borel subgroup of $\mathrm{Sp}_{2 n+1}$ of upper triangular matrices in the odd symplectic basis $\left\{e_{0}, \ldots, e_{2 n}\right\}$, ie the subgroup preserving the flag $\left(E_{1} \subset \cdots \subset\right.$ $\left.E_{2 n+1}\right)$. From 4.10 it follows that the Schubert cells of $G_{\omega}(k, E)$ are $B$-stable. We actually have:

Proposition 4.13. The Schubert cells of $G_{\omega}(k, E)$ are the orbits of $B$.

Proof. The Schubert cells of $G_{\omega}(k, E)$ are orbits of the Borel subgroup $B_{2 n+2}$ of $\mathrm{Sp}_{2 n+2}$ of upper triangular matrices in the symplectic basis $\left\{e_{0}, \ldots, e_{2 n+1}\right\}$. But $B_{2 n+2}$ acts on the Schubert subvariety $X_{\max }$ through the restriction morphism $\left.b \mapsto b\right|_{E}$, and the image of $B_{2 n+2}$ via this morphism is $B$.

4.14. We equip $\mathbf{I}_{k, 2 n+1}^{\omega}$ with the order relation for which $I \leqslant J$ if and only if $i_{\alpha} \leqslant j_{\alpha}$ for all $1 \leqslant i \leqslant k$. This corresponds with the order on $\mathbf{I}_{k, 2 n+2}^{\omega}$ when we identify $\mathbf{I}_{k, 2 n+1}^{\omega}$ with the interval $\left\{I \in \mathbf{I}_{k, 2 n+2}^{\omega} \mid I \leqslant \mathbf{m a x}\right\}$. It follows that the order on $\mathbf{I}_{k, 2 n+1}^{\omega}$ describes the Bruhat order on the set of Schubert cells of $G_{\omega}(k, E)$, that is, for all $I, J \in \mathbf{I}_{k, 2 n+1}^{\omega}$,

$$
C_{I} \subset \overline{C_{J}} \Longleftrightarrow I \leqslant J
$$

4.15. Define the Schubert subvarieties of the odd symplectic grassmannian $G_{\omega}(k, E)$ to be the closures of the Schubert cells. Since the Bruhat order on the set of Schubert cells agrees with the order on $\mathbf{I}_{k, 2 n+1}^{\omega}$, the Schubert subvariety $X_{I}=\overline{C_{I}}$ verifies

$$
X_{I}=\bigcup_{J \leqslant I} C_{J} .
$$

Therefore, in terms of the incidence with the flag $\left(E_{1} \subset \cdots \subset E_{2 n+1}\right)$, the Schubert subvariety $X_{I}$ is characterized by

$$
X_{I}=\left\{V \in G_{\omega}(k, E) \mid \operatorname{dim}\left(V \cap E_{i_{\alpha}}\right) \geqslant \alpha, \quad 1 \leqslant \alpha \leqslant k\right\} .
$$

4.16. The Picard group of the odd symplectic grassmannian $G_{\omega}(k, E)$ is $\mathbb{Z}$. Indeed, since $G_{\omega}(k, E)$ has a cellular decomposition, the Picard group coincides with the free abelian group generated by the classes of the closures of the codimension 1 cells. In $G_{\omega}(k, E)$ there is only one codimension 1 cell, ie only one Schubert divisor. We can easily check that, for $1 \leqslant k \leqslant n$, the Schubert divisor is

$$
X_{\overline{k+1}} \overline{k-1} \ldots \overline{2} \overline{1}=\left\{V \in G_{\omega}(k, E) \mid V \cap E_{2 n-k+1} \neq 0\right\}
$$

and, for $k=n+1$, it is

$$
X_{0 n \overline{n-1} . . . \overline{2} \overline{1}}=\left\{V \in G_{\omega}(n+1, E) \mid \operatorname{dim}\left(V \cap E_{n+1}\right) \geqslant 2\right\} .
$$

4.17. Schubert cells in the odd symplectic flag manifold $\mathbb{F}_{\omega}(2 n+1)$. In order to define the Schubert cells in the odd symplectic flag manifold $\mathbb{F}_{\omega}(2 n+1)$, we proceed, as in the case of the odd symplectic grassmannians, by first identifying $\mathbb{F}_{\omega}(2 n+1)$ to a Schubert subvariety in a symplectic flag manifold $\mathbb{F}_{\omega}(2 n+2)$. With the same notations as in 4.8 a flag $V_{\bullet} \in \mathbb{F}_{\omega}(E)$ is the same thing as a flag $V_{\bullet} \in \mathbb{F}_{\tilde{\omega}}(\tilde{E})$ for which $V_{n+1} \subset E$, that is, $\mathbb{F}_{\omega}(E)$ identifies with the subvariety

$$
\left\{\left(V_{1} \subset \cdots \subset V_{n+1}\right) \in \mathbb{F}_{\tilde{\omega}}(\tilde{E}) \mid V_{n+1} \subset E\right\} .
$$


This is a Schubert subvariety in $\mathbb{F}_{\tilde{\omega}}(\tilde{E})$. With respect to the flag $\left(E_{1} \subset \cdots \subset\right.$ $E_{2 n+2}$ ), and in the notation of 2.14 this is the Schubert subvariety $X_{\overline{1} \overline{2} \ldots \bar{n} 0}$ associated to the signed permutation $\overline{1} \overline{2} \ldots \bar{n} 0 \in W\left(\mathrm{Sp}_{2 n+2}\right)$.

Identifying then $\mathbb{F}_{\omega}(E)$ with $X_{\overline{1} \overline{2} \ldots .}$, we define the Schubert cells of $\mathbb{F}_{\omega}(E)$ (with respect to the flag $\left(E_{1} \subset \cdots \subset E_{2 n+1}\right)$ ) to be the Schubert cells of $\mathbb{F}_{\tilde{\omega}}(\tilde{E})$ (with respect to the flag $\left.\left(E_{1} \subset \cdots \subset E_{2 n+1} \subset E_{2 n+2}\right)\right)$ which are included in $X_{\overline{1} \overline{2} \ldots \bar{n} 0}$.

The Schubert cells of $\mathbb{F}_{\omega}(E)$ are therefore parametrized by the interval

$$
\left\{w \in W\left(\mathrm{Sp}_{2 n+2}\right) \mid w \leqslant \overline{1} \overline{2} \ldots \bar{n} 0\right\}
$$

in the Weyl group $W\left(\mathrm{Sp}_{2 n+2}\right)$ of $\mathrm{Sp}_{2 n+2}$. We introduce the following notation:

Notation 4.18. We denote the interval $\left\{w \in W\left(\operatorname{Sp}_{2 n+2}\right) \mid w \leqslant \overline{1} \overline{2} \ldots \bar{n} 0\right\}$ by $W\left(\mathrm{Sp}_{2 n+1}\right)$.

This notation is somewhat misleading since it might suggest that $W\left(\mathrm{Sp}_{2 n+1}\right)$ is the Weyl group of $\mathrm{Sp}_{2 n+1}$. Of course, $W\left(\mathrm{Sp}_{2 n+1}\right)$ is not a group at all, and the Weyl group of $\mathrm{Sp}_{2 n+1}$ coincides with the Weyl group of $\mathrm{Sp}_{2 n}$.

Proposition 4.19. The elements of $W\left(\mathrm{Sp}_{2 n+1}\right)$ are precisely those signed permutations which in the one-line notation do not contain $\overline{0}$, ie those $w$ for which $\overline{0} \notin\{w(0), w(1), \ldots, w(n)\}$.

Proof. Let $w \in W\left(\mathrm{Sp}_{2 n+1}\right)$. We have $w \leqslant \overline{1} \overline{2} \ldots \bar{n} 0$ if and only if the Schubert cell $C_{w}$ is contained in $X_{\overline{1} \overline{2} \ldots \bar{n} 0}$ which is equivalent to $E_{\bullet}^{w} \in X_{\overline{1} \overline{2} \ldots \bar{n} 0}$, where $E_{\bullet}^{w} \in C_{w}$ is the flag generated by the basis $\left\{e_{w(0)}, e_{w(1)}, \ldots, e_{w(n)}\right\}$. By the definition of the Schubert subvariety $X_{\overline{1} \overline{2}} \ldots \bar{n} 0$ this is equivalent to $w(i) \in\{0,1, \ldots, 2 n\}$ for all $0 \leqslant i \leqslant n$ and we are done.

4.20. We derive now the characterization of the Schubert cells by incidence conditions with respect to the flag $\left(E_{1} \subset \cdots \subset E_{2 n+1}\right)$. Let us first note that if $w \in W\left(\mathrm{Sp}_{2 n+1}\right) \subset W\left(\mathrm{Sp}_{2 n+2}\right)$ then, by the last proposition, the rank function $r_{w}$ of $w$ is determined by its restriction to the rectangle $\{0, \ldots, n\} \times\{0, \ldots, 2 n\}$.

Proposition 4.21. The Schubert cell $C_{w}$ of $\mathbb{F}_{\omega}(E)$ associated to $w \in W\left(\mathrm{Sp}_{2 n+1}\right)$ is the set of flags of isotropic subspaces $V_{\bullet}=\left(V_{1} \subset \cdots \subset V_{n+1}\right)$ which verify

$$
\operatorname{dim}\left(V_{i} \cap E_{j}\right)=r_{w}(i, j)
$$

for all $0 \leqslant i \leqslant n$ and $0 \leqslant j \leqslant 2 n$.

Proof. These conditions are obtained from those defining $C_{w}$ in $\mathbb{F}_{\tilde{\omega}}(\tilde{E})$ by forgetting the case $j=2 n+1$ for which the conditions are automatically verified since $V_{n+1} \subset$ $E$ and $w \in W\left(\operatorname{Sp}_{2 n+1}\right) \subset W\left(\operatorname{Sp}_{2 n+2}\right)$.

4.22. Analogously to the symplectic case, the Schubert cell $C_{w}$ in $\mathbb{F}_{\omega}(E)$ is the set of flags of isotropic subspaces which, with respect to the odd symplectic basis $\left\{e_{0}, \ldots, e_{2 n}\right\}$, have an adapted basis given by the rows of a $(n+1) \times(2 n+1)$ matrix $\left(a_{i j}\right)$ for which $a_{i w(i)}=1$ and $a_{i j}=0$ for all $0 \leqslant i \leqslant n$ and $0 \leqslant j \leqslant 2 n, j>w(i)$.

4.23. From 4.21 it follows that the Schubert cells of $\mathbb{F}_{\omega}(E)$ are stable under the Borel subgroup $B$ of $\operatorname{Sp}_{2 n+1}$ which preserves the flag $\left(E_{1} \subset \cdots \subset E_{2 n+1}\right)$. We actually have:

Proposition 4.24. The Schubert cells of $\mathbb{F}_{\omega}(E)$ are the orbits of $B$.

Proof. The Schubert cells of $\mathbb{F}_{\omega}(E)$ are orbits of the Borel subgroup $B_{2 n+2}$ of the symplectic group $\operatorname{Sp}_{2 n+2}$ which preserves the flag $\left(E_{1} \subset \cdots \subset E_{2 n+1} \subset E_{2 n+2}\right)$. The assertion follows since $B_{2 n+2}$ acts on $X_{\overline{1} \overline{2} \ldots \bar{n} 0}$ trough the restriction morphism $\left.b \mapsto b\right|_{E}$ and the image of $B_{2 n+2}$ via this morphism is $B$. 
4.25. We equip the subset $W\left(\mathrm{Sp}_{2 n+1}\right) \subset W\left(\mathrm{Sp}_{2 n+2}\right)$ with the order induced by the Bruhat order of the Weyl group $W\left(\mathrm{Sp}_{2 n+2}\right)$. In this way, the order on $W\left(\mathrm{Sp}_{2 n+1}\right)$ describes the Bruhat order on the set of Schubert cells of $\mathbb{F}_{\omega}(E)$, that is, for all $w, w^{\prime} \in W\left(\mathrm{Sp}_{2 n+1}\right)$,

$$
C_{w} \subset \overline{C_{w^{\prime}}} \Longleftrightarrow w \leqslant w^{\prime} .
$$

Define the Schubert subvarieties of the odd symplectic flag manifold $\mathbb{F}_{\omega}(E)$ to be the closures of the Schubert cells. For $w \in W\left(\mathrm{Sp}_{2 n+1}\right)$, the Schubert subvariety $X_{w}=\overline{C_{w}}$ is then given by

$$
X_{w}=\bigcup_{w^{\prime} \leqslant w} C_{w^{\prime}} .
$$

In terms of the incidence with the flag $\left(E_{1} \subset \cdots \subset E_{2 n+1}\right)$, the Schubert subvariety $X_{w}$ is the set of flags of isotropic subspaces $V_{\bullet}=\left(V_{1} \subset \cdots \subset V_{n+1}\right)$ which verify

$$
\operatorname{dim}\left(V_{i} \cap E_{j}\right) \geqslant r_{w}(i, j)
$$

for all $0 \leqslant i \leqslant n$ and $0 \leqslant j \leqslant 2 n$.

4.26. We can count the Schubert cells of $\mathbb{F}_{\omega}(E)$ of a given dimension of and thus compute the Poincaré polynomial of $\mathbb{F}_{\omega}(E)$. We have:

Proposition 4.27. The Poincaré polynomial of the variety $\mathbb{F}_{\omega}(E)$ is

$$
P\left(\mathbb{F}_{\omega}(E), q\right)=\frac{\left(q^{n+1}-1\right)\left(q^{2 n}-1\right)\left(q^{2 n-2}-1\right) \ldots\left(q^{2}-1\right)}{(q-1)^{n+1}} .
$$

Since $2,4, \ldots, 2 n, n+1$ are the exponents of the Weyl group $W\left(\mathrm{D}_{n+1}\right)$ of type $\mathrm{D}_{n+1}$ (cf. Bou68), it follows that (7) coincides with the Poincare polynomial of the flag variety $G / B$ for $G$ of type $\mathrm{D}_{n+1}$. We prove this proposition in Mih where we also show that $\mathbb{F}_{\omega}(E)$ and $G / B$ with $G$ of type $\mathrm{D}_{n+1}$ have actually the same singular cohomology rings.

\section{THE AUTOMORPHISM GROUP OF AN ODD SYMPLECTIC GRASSMANNIAN}

We compute here the automorphism group of the odd symplectic grassmannian $G_{\omega}(k, E)$. If $k=1$ then $G_{\omega}(1, E)$ is just the projective space $\mathbb{P} E$ and its automorphism group is $\mathrm{PSL}_{2 n+1}$. If $k=n+1$ then, as seen in 4.2 the odd symplectic grassmannian $G_{\omega}(n+1, E)$ is isomorphic to the symplectic grassmannian $G_{\omega}(n, F)$, and so its automorphism group is $\mathrm{PSp}_{2 n}$. For the remaining cases we have the following result:

Proposition 5.1. For $2 \leqslant k \leqslant n$, the automorphism group of the odd symplectic grassmannian $G_{\omega}(k, 2 n+1)$ is $\mathrm{PSp}_{2 n+1}=\mathrm{Sp}_{2 n+1} /\{ \pm 1\}$.

The proof we will present here is inspired from a now standard method of computing the automorphism group of the usual grassmannian (see for example Har95 $10.19])$ and relies on an analysis of the linear spaces contained in $G_{\omega}(k, E)$.

But before filling in the details, let us mention that there is a different, quite straightforward way to compute the Lie algebra of this automorphism group. This method is detailed in Mih05 §2.3.1] and roughly consists of the following steps. First of all, the Lie algebra of the automorphism group of $G_{\omega}(k, E)$ coincides with the space of global sections of the tangent bundle $H^{0}\left(G_{\omega}(k, E), T G_{\omega}(k, E)\right.$ ) (cf. Akh95, §2.3]). In order to compute this we use the description of $G_{\omega}(k, E)$ as the zero locus of $\omega$ seen as a generic section of the bundle $\Lambda^{2} T^{*}$ on the grassmannian $G(k, E)$. Then the differential of this section identifies $\Lambda^{2} T^{*}$ with the normal bundle of $G_{\omega}(k, E)$ in $G(k, E)$, so that the space of global sections we want to compute is the kernel of the map

$$
H^{0}\left(G_{\omega}(k, E),\left.T G(k, E)\right|_{G_{\omega}(k, E)}\right) \stackrel{d \omega}{\longrightarrow} H^{0}\left(G_{\omega}(k, E),\left.\Lambda^{2} T^{*}\right|_{G_{\omega}(k, E)}\right) .
$$


We now use the Koszul complex associated to the section $\omega$ to derive resolutions by locally free sheaves on the grassmannian $G(k, E)$ for the two restrictions $\left.T G(k, E)\right|_{G_{\omega}(k, E)}$ and $\left.\Lambda^{2} T^{*}\right|_{G_{\omega}(k, E)}$. With the help of Bott's theorem we prove that these resolutions are acyclic, so that taking the global sections yields resolutions for the two spaces in (8). Specifically, (8) becomes

$$
\mathfrak{s l}(E) \longrightarrow \Lambda^{2} E^{*} / \omega
$$

where the map is sending an element $X \in \mathfrak{s l}(E)$ to the image in $\Lambda^{2} E^{*} / \omega$ of the transform $X \cdot \omega$ corresponding to the $\mathfrak{s l}(E)$-module structure of $\Lambda^{2} E^{*}$. The kernel of (9) is then the Lie algebra $\{X \in \mathfrak{s l}(E) \mid X \cdot \omega \in \mathbb{C} \omega\}$ which identifies with $\{X \in \mathfrak{g l}(E) \mid X \cdot \omega \in \mathbb{C} \omega\} / \mathbb{C I d}$ and so with $\mathfrak{s p}_{2 n+1}=\{X \in \mathfrak{g l}(E) \mid X \cdot \omega=0\}$ (as Lie algebras, and not only as vector spaces, since Id is central in $\mathfrak{g l}(E))$. The Lie algebra of the automorphism group of $G_{\omega}(k, E)$ is therefore $\mathfrak{s p}_{2 n+1}$.

5.2. We now proceed with our proof of proposition [5.1] The outline of the proof is the following. First we show that, in its Plücker embedding via the inclusion $G_{\omega}(k, E) \subset G(k, E)$, all the automorphisms of $G_{\omega}(k, E)$ come from automorphisms of the ambient projective space. This implies in particular that any automorphism of $G_{\omega}(k, E)$ induces automorphisms of the Fano schemes of linear spaces contained in $G_{\omega}(k, E)$. It turns out that a certain Fano scheme of $G_{\omega}(k, E)$ is either irreducible or has two non-isomorphic irreducible components, and in both cases one of its irreducible components is isomorphic to the blow-up of $G_{\omega}(k-1, E)$ along its closed $\mathrm{Sp}_{2 n+1}$-orbit. We show then that any automorphism of this Fano scheme coming from an automorphism of $G_{\omega}(k, E)$ induces further an automorphism of $G_{\omega}(k-$ $1, E)$. Iterating this, any automorphism of $G_{\omega}(k, E)$ induces an automorphism of $G_{\omega}(1, E)=\mathbb{P} E$, that is, an element of $\mathrm{PSL}_{2 n+1}$, which, as it comes from an automorphism of $G_{\omega}(2, E)$, will be shown to be in $\mathrm{PSp}_{2 n+1}$. Finally, we show that the initial automorphism of $G_{\omega}(k, E)$ coincides with the automorphism induced by this element of $\mathrm{PSp}_{2 n+1}$.

5.3. Let's start with some preliminaries. We first recall Bott's theorem in the particular case of the grassmannian $G(k, E)$ which will be used below. Let $P_{k} \subset$ $\mathrm{SL}_{2 n+1}$ be the parabolic subgroup which preserves the space generated by the last $k$ vectors of the basis $\left\{e_{0}, \ldots, e_{2 n}\right\}$, so that $G(k, E)=\mathrm{SL}_{2 n+1} / P_{k}$. In $\mathrm{SL}_{2 n+1}$ we fix the maximal torus of diagonal matrices in the basis $\left\{e_{0}, \ldots, e_{2 n}\right\}$ and the Borel subgroup of upper triangular matrices in the same basis; the parabolic $P_{k}$ contains then the opposite Borel subgroup. The weights of $\mathrm{SL}_{2 n+1}$ identify with the tuples $\lambda=\left(\lambda_{0}, \ldots, \lambda_{2 n}\right)$ modulo shifts $\left(\lambda_{0}, \ldots, \lambda_{2 n}\right) \mapsto\left(\lambda_{0}+a, \ldots, \lambda_{2 n}+a\right)$. A weight $\lambda=\left(\lambda_{0}, \ldots, \lambda_{2 n}\right)$ is singular if it has two equal parts and dominant if $\lambda_{0} \geqslant \cdots \geqslant \lambda_{2 n}$. In the latter case, if the $\lambda_{i}$ are non negative, we see $\lambda$ as a partition and denote $|\lambda|=\sum \lambda_{i}$ its weight and $\ell(\lambda)$, the number of non zero parts, its length. For $\lambda$ dominant, the simple $\mathrm{SL}_{2 n+1}$-module with highest weight $\lambda$ is isomorphic to the Schur power $S_{\lambda} E$.

Irreducible homogeneous vector bundles on $G(k, E)$ are determined by irreducible $P_{k}$-modules, which in turn are determined by $P_{k}$-dominant weights, that is weights $\lambda=\left(\lambda_{0}, \ldots, \lambda_{2 n}\right)$ such that $\lambda_{0} \geqslant \cdots \geqslant \lambda_{2 n-k}$ and $\lambda_{2 n-k+1} \geqslant \cdots \geqslant$ $\lambda_{2 n}$. If $T$ and $Q$ denote the tautological, respectively the quotient, bundles on the grassmannian $G(k, E)$, whose fibers at a point $V \in G(k, E)$ are $V$, respectively $E / V$, then the irreducible bundle corresponding to a $P_{k}$-dominant weight $\lambda=\left(\lambda_{0}, \ldots, \lambda_{2 n}\right)$ is given in terms of Schur powers of $T$ and $Q$ by

$$
E_{\lambda}=S_{\lambda^{\prime}} Q \otimes S_{\lambda^{\prime \prime}} T
$$

where $\lambda^{\prime}=\left(\lambda_{0} \geqslant \cdots \geqslant \lambda_{2 n-k}\right)$ and $\lambda^{\prime \prime}=\left(\lambda_{2 n-k+1} \geqslant \cdots \geqslant \lambda_{2 n}\right)$. 
The Weyl group of $\mathrm{SL}_{2 n+1}$ is isomorphic to the permutation group $S_{2 n+1}$ and acts on weights by $w \cdot\left(\lambda_{0}, \ldots, \lambda_{2 n}\right)=\left(\lambda_{w^{-1}(0)}, \ldots, \lambda_{w^{-1}(2 n)}\right)$. Let $\rho=(2 n+1,2 n, \ldots, 1)$ denote the smallest strictly decreasing partition, which modulo a shift coincides with the half-sum of the positive roots. For $\lambda$ a $P_{k}$-dominant weight, the cohomology of the associated irreducible bundle $E_{\lambda}$ is described by Bott's theorem:

Theorem. If $\lambda+\rho$ is singular then $H^{i}\left(G(k, E), E_{\lambda}\right)=0$ for all $i$. If not, let $w \in S_{2 n+1}$ be the unique permutation such that $w(\lambda+\rho)$ is dominant, and let $\ell$ be its length. Then $H^{\ell}\left(G(k, E), E_{\lambda}\right)$ is the simple $\mathrm{SL}_{2 n+1}$-module of highest weight $w(\lambda+\rho)-\rho$ and $H^{i}\left(G(k, E), E_{\lambda}\right)=0$ for all $i \neq \ell$.

We'll also need the plethysm formula for the decomposition of $\Lambda^{j}\left(\Lambda^{2} V\right)$ as a $\mathrm{GL}(V)$-module. Recall first that the Frobenius notation $\lambda=\left(a_{1}, \ldots, a_{r} \mid b_{1}, \ldots, b_{r}\right)$ for a partition $\lambda$ encodes its hook decomposition, that is, in the Ferrers diagram of $\lambda$ there are $a_{i}$ boxes to the right of the $i$-th box on the diagonal and $b_{i}$ boxes below. The rank $r$ of $\lambda$ is the number of boxes on the diagonal. We have (cf. Mac95])

$$
\Lambda^{i}\left(\Lambda^{2} V\right)=\bigoplus_{|\lambda|=i} S_{\lambda^{-}} V
$$

where the sum is indexed by all strictly decreasing partitions of weight $i$ and for a strictly decreasing partition $\lambda$ we denote

$$
\lambda^{-}=\left(\lambda_{1}-1, \ldots, \lambda_{\ell}-1 \mid \lambda_{1}, \ldots, \lambda_{\ell}\right)
$$

5.4. We embed $G_{\omega}(k, E)$ in $G(k, E)$ and further, via the Plücker embedding of $G(k, E)$, in $\mathbb{P} \Lambda^{k} E$. We set out to prove that any automorphism of $G_{\omega}(k, E)$ comes from an automorphism of the ambient projective space $\mathbb{P} \Lambda^{k} E$.

Proposition 5.5. The embedding $G_{\omega}(k, E) \subset \mathbb{P} \Lambda^{k} E$ is linearly normal, that is, the restriction morphism

$$
H^{0}\left(\mathbb{P} \Lambda^{k} E, \mathcal{O}_{\mathbb{P} \Lambda^{k} E}(1)\right) \longrightarrow H^{0}\left(G_{\omega}(k, E), \mathcal{O}_{G_{\omega}(k, E)}(1)\right)
$$

is surjective.

Proof. To simplify the notation, we write $G$ instead of $G(k, E)$ and $G_{\omega}$ instead of $G_{\omega}(k, E)$. The line bundle $\mathcal{O}(1)$ on $G$ corresponding to the Plücker embedding is isomorphic to $\operatorname{det} T^{*}$. Since $H^{0}\left(G, \operatorname{det} T^{*}\right)=\Lambda^{k} E^{*}$, it suffices to show that the restriction morphism

$$
H^{0}\left(G, \mathcal{O}_{G}(1)\right) \longrightarrow H^{0}\left(G_{\omega}, \mathcal{O}_{G_{\omega}}(1)\right)
$$

is surjective. We regard $G_{\omega}$ as the zero locus of $\omega$ seen as a section of the bundle $\Lambda^{2} T^{*}$ on $G$. We consider the Koszul complex of $\omega$ which we twist by $\operatorname{det} T^{*}$ and obtain the exact complex

$$
\begin{aligned}
0 \longrightarrow \Lambda^{r}\left(\Lambda^{2} T\right) \otimes \operatorname{det} T^{*} & \longrightarrow \cdots \longrightarrow \Lambda^{2}\left(\Lambda^{2} T\right) \otimes \operatorname{det} T^{*} \longrightarrow \\
& \left.\longrightarrow \Lambda^{2} T \otimes \operatorname{det} T^{*} \longrightarrow \operatorname{det} T^{*} \longrightarrow \operatorname{det} T^{*}\right|_{G_{\omega}} \longrightarrow 0
\end{aligned}
$$

where $r=\frac{1}{2} k(k-1)$. To obtain the surjectivity of (11) it suffices to show that this complex is acyclic, ie that the bundles in (12) have no higher cohomology (this follows in a standard way by chopping (12) into short exact sequences and using the corresponding cohomology long exact sequences). By (10), we have

$$
\Lambda^{j}\left(\Lambda^{2} T\right) \otimes \operatorname{det} T^{*}=\bigoplus_{|\lambda|=j} S_{\lambda^{-}} T \otimes \operatorname{det} T^{*}
$$

so any irreducible component of $\Lambda^{j}\left(\Lambda^{2} T\right) \otimes \operatorname{det} T^{*}$ is of the form $E_{\eta}=S_{\mu} T$ where $\mu=\left(\mu_{1} \geqslant \cdots \geqslant \mu_{k}\right)$ is the skew partition $\left(\lambda_{1}^{-}-1, \ldots, \lambda_{k}^{-}-1\right)$ for some strictly 
decreasing partition $\lambda$ of weight $j$. Then $\eta=\left(0,0, \ldots, 0, \mu_{1}, \ldots, \mu_{k}\right)$ and

$$
\eta+\rho=\left(2 n+1,2 n, \ldots, k+1, \mu_{1}+k, \ldots, \mu_{k}+1\right) .
$$

Consider first the case $j \geqslant 2$. By construction, we have $\ell\left(\lambda^{-}\right)=\lambda_{1}+1$ and $\lambda_{1}^{-}=\lambda_{1}$. As $T$ is of rank $k, \ell\left(\lambda^{-}\right) \leqslant k$, since otherwise $S_{\lambda^{-}} T=0$, so $\lambda_{1} \leqslant k-1$. Therefore $\mu_{1}+k=\lambda_{1}-1+k \leqslant 2 k-2 \leqslant 2 n+1$. On the other hand, since $|\lambda|=j \geqslant 2$ and $\lambda$ is strictly decreasing, we have $\lambda_{1} \geqslant 2$ and so $\mu_{1}+k=\lambda_{1}-1+k \geqslant k+1$. The partition $\eta+\rho$ has therefore two equal parts and so is singular. By Bott's theorem, it follows that

$$
H^{i}\left(G, \Lambda^{j}\left(\Lambda^{2} T\right) \otimes \operatorname{det} T^{*}\right)=0 \text { for all } i .
$$

For $j=1$, the bundle $\Lambda^{2} T \otimes \operatorname{det} T^{*}=\Lambda^{k-2} T^{*}$ is given by a dominant weight and therefore has no higher cohomology. Similarly for $j=0$ and the bundle $\operatorname{det} T^{*}$, and we are done.

Proposition 5.6. Any automorphism of the odd symplectic grassmannian $G_{\omega}(k, E)$ is induced by an automorphism of the ambient projective space $\mathbb{P} \Lambda^{k} E$.

Proof. This follows by a standard argument (as in Har77 Example 7.1.1], for example) from the fact that $G_{\omega}(k, E)$ is linearly normal in $\mathbb{P} \Lambda^{k} E$ and its Picard group is $\mathbb{Z}$.

5.7. Denote by $\Lambda^{\langle k\rangle} E$ the kernel of the contraction $\left\llcorner\omega: \Lambda^{k} E \longrightarrow \Lambda^{k-2} E\right.$. Since $H^{0}\left(G, \Lambda^{k-2} T^{*}\right)=\Lambda^{k-2} E^{*}$, taking global sections in (12) and using the vanishings from the proof of 5.5 we get the exact sequence

$$
0 \longrightarrow \Lambda^{k-2} E^{*} \stackrel{\wedge \omega}{\longrightarrow} \Lambda^{k} E^{*} \longrightarrow H^{0}\left(G_{\omega}, \mathcal{O}_{G_{\omega}}(1)\right) \longrightarrow 0 .
$$

Taking the duals, we obtain $H^{0}\left(G_{\omega}, \mathcal{O}_{G_{\omega}}(1)\right)^{*}=\Lambda^{\langle k\rangle} E$ so $G_{\omega}(k, E)$ embeds in the projective subspace $\mathbb{P} \Lambda^{\langle k\rangle} E \subset \mathbb{P} \Lambda^{k} E$ as a non-degenerate subvariety. We actually have:

Proposition 5.8. $G_{\omega}(k, E)=G(k, E) \cap \mathbb{P} \Lambda^{\langle k\rangle} E$.

Proof. Let $V \in G(k, E)$ and $\left\{v_{1}, \ldots, v_{k}\right\}$ a basis of $V$. The contraction of $v_{1} \wedge \cdots \wedge v_{k}$ by $\omega$ is explicitly given by

$$
\left(v_{1} \wedge \cdots \wedge v_{k}\right)\left\llcorner\omega=\sum_{1 \leqslant i<j \leqslant k}(-1)^{i+j} \omega\left(v_{i}, v_{j}\right) v_{1} \wedge \cdots \wedge \widehat{v}_{i} \wedge \cdots \wedge \widehat{v}_{j} \wedge \cdots \wedge v_{k}\right.
$$

so $v_{1} \wedge \cdots \wedge v_{k}$ is in the kernel of $\llcorner\omega$ if and only if $V$ is isotropic.

Note, though, that the intersection in 5.8 is not transverse. Let us denote by $\operatorname{Aut}\left(\mathbb{P} \Lambda^{\langle k\rangle} E, G_{\omega}(k, E)\right)$ the group of automorphisms of the projective space $\mathbb{P} \Lambda^{\langle k\rangle} E$ which preserve $G_{\omega}(k, E)$. We have natural morphisms

$$
\operatorname{PSp}_{2 n+1} \longrightarrow \operatorname{Aut}\left(\mathbb{P} \Lambda^{\langle k\rangle} E, G_{\omega}(k, E)\right) \longrightarrow \operatorname{Aut}\left(G_{\omega}(k, E)\right) .
$$

The second morphism is an isomorphism, since it is surjective by [5.6] and injective since $G_{\omega}(k, E)$ is non-degenerate in $\mathbb{P} \Lambda^{\langle k\rangle} E$. The first morphism is injective, since any line in $E$ is recovered as the intersection of all the $k$-dimensional isotropic subspaces which contain it. Therefore, to conclude our proof of [5.1] it suffices to show that the first morphism is surjective.

We start analyzing the linear spaces contained in $G_{\omega}(k, E)$. We recall first the description we have in the case of the grassmannian $G(k, E)$ (cf. Har95, 6.9]):

Proposition 5.9. A maximal linear space contained in the grassmannian $G(k, E)$ is either of the form

$$
\left\{V \in G(k, E) \mid V \supset V^{k-1}\right\}
$$


for some subspace $V^{k-1} \subset E$ of dimension $k-1$, or of the form

$$
\left\{V \in G(k, E) \mid V \subset V^{k+1}\right\}
$$

for some subspace $V^{k+1} \subset E$ of dimension $k+1$.

For the odd symplectic grassmannian $G_{\omega}(k, E)$ we have:

Proposition 5.10. The maximal linear spaces contained in $G_{\omega}(k, E)$ are of one of the following two types:

$$
\text { type I: } \quad\left\{V \in G_{\omega}(k, E) \mid V^{k-1} \subset V \subset\left(V^{k-1}\right)^{\perp}\right\}= \begin{cases}\mathbb{P}^{2(n-k)+2} & \text { if } V^{k-1} \not \supset e_{0} \\ \mathbb{P}^{2(n-k)+3} & \text { if } V^{k-1} \ni e_{0}\end{cases}
$$

for an isotropic subspace $V^{k-1} \subset E$ of dimension $k-1$,

$$
\text { type II: } \quad\left\{V \in G_{\omega}(k, E) \mid V \subset V^{k+1}\right\}= \begin{cases}\mathbb{P}^{k} & \text { if } V^{k+1} \text { isotropic } \\ \mathbb{P}^{1} & \text { if } \operatorname{rank}\left(\left.\omega\right|_{V^{k+1}}\right)=2\end{cases}
$$

for a subspace $V^{k+1} \subset E$ of dimension $k+1$.

Proof. Since the odd symplectic grassmannian $G_{\omega}(k, E)$ is cut in $G(k, E)$ by a linear space (cf. [5.8), the maximal linear spaces contained in $G_{\omega}(k, E)$ are simply the intersections of $G_{\omega}(k, E)$ with the maximal linear spaces contained in $G(k, E)$.

Let $L$ be a maximal linear space contained in $G(k, E)$ of the first kind described in [5.9] that is, $L=\left\{V \in G(k, E) \mid V \supset V^{k-1}\right\}$ for some subspace $V^{k-1} \subset E$ of dimension $k-1$. If $V^{k-1}$ is not isotropic then $L \cap G_{\omega}(k, E)=\emptyset$. Suppose that $V^{k-1}$ is isotropic and let $V$ be a point of $L$. If $V$ is isotropic then $V \subset\left(V^{k-1}\right)^{\perp}$ and, conversely, if $V \subset\left(V^{k-1}\right)^{\perp}$ then $V$ is isotropic, so

$$
L \cap G_{\omega}(k, E)=\left\{V \in G(k, E) \mid V^{k-1} \subset V \subset\left(V^{k-1}\right)^{\perp}\right\} \simeq \mathbb{P}\left(\left(V^{k-1}\right)^{\perp} / V^{k-1}\right) .
$$

If $V^{k-1}$ does not contain the kernel $R$ of $\omega$ then $\operatorname{dim}\left(V^{k-1}\right)^{\perp}=2 n-k+2$ and $\mathbb{P}\left(\left(V^{k-1}\right)^{\perp} / V^{k-1}\right) \simeq \mathbb{P}^{2 n-2 k+2}$. If $V^{k-1}$ contains $R$ then $\operatorname{dim}\left(V^{k-1}\right)^{\perp}=2 n-k+3$ and $\mathbb{P}\left(\left(V^{k-1}\right)^{\perp} / V^{k-1}\right) \simeq \mathbb{P}^{2 n-2 k+3}$.

Let now $L$ be a maximal linear space contained in $G(k, E)$ of the second kind described in 5.9 that is, $L=\left\{V \in G(k, E) \mid V \subset V^{k+1}\right\}$ for some subspace $V^{k+1} \subset$ $E$ of dimension $k+1$. The intersection $L \cap G_{\omega}(k, E)$ is then the grassmannian of isotropic hyperplanes in $V^{k+1}$. By the lemma below we have three possibilities, according to the rank of $\left.\omega\right|_{V^{k+1}}$ : If $V^{k+1}$ is isotropic then

$$
L \cap G_{\omega}(k, E)=L=\left\{V \in G(k, E) \mid V \subset V^{k+1}\right\} \simeq \mathbb{P}^{k} ;
$$

If $\operatorname{rank}\left(\left.\omega\right|_{V^{k+1}}\right)=2$ then

$$
L \cap G_{\omega}(k, E)=\left\{V \in G(k, E) \mid \operatorname{Ker}\left(\left.\omega\right|_{V^{k+1}}\right) \subset V \subset V^{k+1}\right\} \simeq \mathbb{P}^{1} ;
$$

If $\operatorname{rank}\left(\left.\omega\right|_{V^{k+1}}\right)>2$ then $L \cap G_{\omega}(k, E)=\emptyset$. This concludes the proof.

Lemma 5.11. Let $W$ be a space equipped with a non zero skew-form $\omega$. If $\operatorname{rank} \omega=$ 2 then the isotropic hyperplanes of $W$ are precisely those which contain $\operatorname{Ker} \omega$. If rank $\omega>2$ then there is no isotropic hyperplane in $W$.

Proof. Denote still by $\omega$ the canonical morphism $\omega: W \rightarrow W^{*}$. We write $\perp_{\omega}$ to denote the orthogonal with respect to $\omega$ and $\perp$ to denote the orthogonal with respect to the duality $W \otimes W^{*} \rightarrow \mathbb{C}$. Let $H \subset W$ be a hyperplane. Then

$$
H \text { isotropic } \Longleftrightarrow H \subset H^{\perp_{\omega}}=\omega(H)^{\perp} \Longleftrightarrow H^{\perp} \supset \omega(H) \text {. }
$$

Since $\operatorname{dim} H^{\perp}=1$, this is equivalent to $\omega(H)=0$ or $\omega(H)=H^{\perp}$. We cannot have $\omega(H)=0$ since Ker $\omega$ is of codimension at least 2. If $\omega(H)=H^{\perp}$ then Ker $\omega \cap H$ is a hyperplane of $H$ so codim $\operatorname{Ker} \omega \leqslant 2$, and so codim $\operatorname{Ker} \omega=2$ and $\operatorname{Ker} \omega \subset H$. 
5.12. The linear spaces contained in $G_{\omega}(k, E)$ which will prove useful in our computation of the automorphism group of $G_{\omega}(k, E)$ are those of dimension $2(n-k)+2$. Recall that for a projective variety $X \subset \mathbb{P}^{N}$ and an integer $\ell \in \mathbb{N}$, the Fano scheme $\mathbf{F}(\ell, X)$ is the subvariety of the grassmannian $\mathbb{G}(\ell, N)$ of $\mathbb{P}^{\ell}$ 's in $\mathbb{P}^{N}$ parametrizing the linear subspaces of $\mathbb{P}^{N}$ of dimension $\ell$ contained in $X$.

In what follows we will focus on the Fano scheme $\mathbf{F}\left(2(n-k)+2, G_{\omega}(k, E)\right)$ of linear spaces of dimension $2(n-k)+2$ contained in $G_{\omega}(k, E)$. For simplicity, we will denote it $\mathbf{F}$.

Proposition 5.13. If $k<\frac{2}{3}(n+1)$ then the Fano scheme $\mathbf{F}$ parametrizing the $\mathbb{P}^{2(n-k)+2}$ 's contained in $G_{\omega}(k, E)$ is irreducible and isomorphic to the variety

$$
\mathbf{F}^{1}=\left\{\left(V^{k-1}, H\right) \in G_{\omega}(k-1, E) \times G(2 n-k+2, E) \mid V^{k-1} \subset H \subset\left(V^{k-1}\right)^{\perp}\right\}
$$

which identifies to the blow-up of $G_{\omega}(k-1, E)$ along its closed $\mathrm{Sp}_{2 n+1}$-orbit.

If $k \geqslant \frac{2}{3}(n+1)$ then $\mathbf{F}$ has two disjoint irreducible components: one is isomorphic to the variety $\mathbf{F}^{1}$ above, the other is isomorphic to the variety

$$
\mathbf{F}^{2}=\left\{\left(W, V^{k+1}\right) \in G(3 k-2(n+1), E) \times G_{\omega}(k+1, E) \mid W \subset V^{k+1}\right\}
$$

and these two components are not isomorphic.

We will prove this proposition in several steps. First, a little piece of terminology. Any $\mathbb{P}^{2(n-k)+2}$ contained in $G_{\omega}(k, E)$ is included in some maximal linear space contained in $G_{\omega}(k, E)$. If the maximal linear space is of type I (according to 5.10) then we will say that the $\mathbb{P}^{2(n-k)+2}$ is of the first kind. Otherwise, ie if the maximal linear space is of type II, we will say the $\mathbb{P}^{2(n-k)+2}$ is of the second kind. Note that this terminology is coherent, that is, a $\mathbb{P}^{2(n-k)+2}$ cannot simultaneously be of the first and of the second kind since the intersection of a type I maximal linear space with a type II maximal linear space is contained in a $\mathbb{P}^{1}$ :

$$
\begin{aligned}
&\left\{V \in G_{\omega}(k, E) \mid V^{k-1} \subset V \subset\left(V^{k-1}\right)^{\perp}\right\} \cap\left\{V \in G_{\omega}(k, E) \mid V \subset V^{k+1}\right\} \subset \\
& \subset\left\{V \in G(k, E) \mid V^{k-1} \subset V \subset V^{k+1}\right\} \simeq \mathbb{P}^{1} .
\end{aligned}
$$

Note also that if $k<2(n-k)+2$, or equivalently $k<\frac{2}{3}(n+1)$, then from the definition it follows that there is no $\mathbb{P}^{2(n-k)+2}$ of the second kind.

Lemma 5.14. The $\mathbb{P}^{2(n-k)+2}$ 's of the first kind are parametrized by the variety $\mathbf{F}^{1}$.

Proof. Consider a $\mathbb{P}^{2(n-k)+2}$ of the first kind. If it is maximal, then according to 5.10 it is determined by giving a $V^{k-1}$ isotropic of dimension $k-1$ which does not contain $e_{0}$. If it is not maximal then it is a hyperplane in a maximal $\mathbb{P}^{2(n-k)+3}$ of type $\mathrm{I}$, ie it is determined by giving a $V^{k-1}$ isotropic of dimension $k-1$ which contains $e_{0}$ and a hyperplane $H$ of $\left(V^{k-1}\right)^{\perp}$ which contains $V^{k-1}$ :

$$
\mathbb{P}^{2(n-k)+2}=\left\{V \in G(k, E) \mid V^{k-1} \subset V \subset H \subset\left(V^{k-1}\right)^{\perp}\right\} .
$$

Now consider a point $\left(V^{k-1}, H\right) \in \mathbf{F}^{1}$. If $V^{k-1} \not \supset e_{0}$ then $\operatorname{dim}\left(V^{k-1}\right)^{\perp}=2 n-k+2$, so $H=\left(V^{k-1}\right)^{\perp}$. If $V^{k-1} \ni e_{0}$ then $\operatorname{dim}\left(V^{k-1}\right)^{\perp}=2 n-k+3$ and so $H$ is a hyperplane in $\left(V^{k-1}\right)^{\perp}$. This shows that any $\mathbb{P}^{2(n-k)+2}$ of the first kind is of the form

$$
\left\{V \in G(k, E) \mid V^{k-1} \subset V \subset H\right\}
$$

for some $\left(V^{k-1}, H\right) \in \mathbf{F}^{1}$. Conversely, any linear space of the form (13) is a $\mathbb{P}^{2(n-k)+2}$ contained in $G_{\omega}(k, E)$, and we are done.

Lemma 5.15. The $\mathbb{P}^{2(n-k)+2}$ 's of the second kind are parametrized by $\mathbf{F}^{2}$. 
Proof. A $\mathbb{P}^{2(n-k)+2}$ of the second kind is included in a maximal $\mathbb{P}^{k}$ of type II, so, according to [5.10 it is defined by giving a $V^{k+1}$ isotropic dimension $k+1$ and a subspace $V^{3 k-2(n+1)} \subset V^{k+1}$ of dimension $3 k-2(n+1)$ :

$$
\mathbb{P}^{2(n-k)+2}=\left\{V \in G(k, E) \mid V^{3 k-2(n+1)} \subset V \subset V^{k+1}\right\} .
$$

Conversely, any linear space of the form (14) is contained in $G_{\omega}(k, E)$.

Lemma 5.16. The projective variety $\mathbf{F}^{1}$ is isomorphic to the blow-up of the odd symplectic grassmannian $G_{\omega}(k-1, E)$ along its closed $\mathrm{Sp}_{2 n+1}$-orbit.

Proof. Let $p: \mathbf{F}^{1} \rightarrow G_{\omega}(k-1, E)$ denote the projection on the first factor, and let $V^{k-1} \in G_{\omega}(k-1, E)$. From the discussion in the proof of lemma 5.14 it follows that if $V^{k-1} \not \supset e_{0}$ then the fiber $p^{-1}\left(V^{k-1}\right)$ reduces to the point $\left(V^{k-1},\left(V^{k-1}\right)^{\perp}\right)$ and if $V^{k-1} \ni e_{0}$ then the fiber $p^{-1}\left(V^{k-1}\right)$ identifies with

$$
\left\{H \mid H \text { hyperplane in }\left(V^{k-1}\right)^{\perp}, H \supset V^{k-1}\right\}=\mathbb{P}\left(\left(V^{k-1}\right)^{\perp} / V^{k-1}\right)^{*} .
$$

So, above the open $\operatorname{Sp}_{2 n+1}$-orbit $X_{1} \subset G_{\omega}(k-1, E)$ the projection $p$ is an isomorphism, and above the closed $\mathrm{Sp}_{2 n+1}$-orbit $X_{0} \subset G_{\omega}(k-1, E)$ it is a fibration with fiber $\mathbb{P}^{2 n-2 k+3}$.

Since $X_{0}$ is of codimension $2 n-2 k+4$ in $G_{\omega}(k-1, E)$ it follows that $p^{-1}\left(X_{0}\right)$ is a divisor in $\mathbf{F}^{1}$, and to prove that $\mathbf{F}^{1}$ is the blow-up of $X_{0}$ in $G_{\omega}(k-1, E)$ it suffices to show that $\mathbf{F}^{1}$ is smooth (cf. GH78. Ch. $\left.4, \S 6\right]$ ). We will show that $\mathbf{F}^{1}$ is the zero locus of a sufficiently generic section of a vector bundle on the partial flag variety

$$
\mathbb{F}(k-1,2 n-k+2, E)=\left\{\left(V^{k-1}, V^{2 n-k+2}\right) \mid V^{k-1} \subset V^{2 n-k+2} \subset E\right\}
$$

(with the convention, implicitly used already, to write dimensions as superscripts). Note that this flag variety is a component of the Fano scheme $\mathbf{F}(2(n-k)+2, G(k, E))$ parametrizing the $\mathbb{P}^{2(n-k)+2}$ 's contained in the grassmannian $G(k, E)$.

For simplicity we denote in what follows $a=k-1$ and $b=2 n-k+2$. Let $T^{a}$ and $T^{b}$ be the tautological bundles on the flag variety $\mathbb{F}(a, b, E)$ whose fibers at a point $V^{\bullet}=\left(V^{a} \subset V^{b}\right) \in \mathbb{F}(a, b, E)$ are $V^{a}$, respectively $V^{b}$. The skew-form $\omega$ defines a section of the bundle $\left(T^{a}\right)^{*} \otimes\left(T^{b}\right)^{*}$ whose value at the point $\left(V^{a} \subset V^{b}\right)$ is the restriction of $\omega$ to the subspace $V^{a} \times V^{b} \subset E \times E$.

A point $\left(V^{a} \subset V^{b}\right)$ of $\mathbb{F}(a, b, E)$ is in $\mathbf{F}^{1}$ if and only if $V^{a}$ is isotropic and $V^{b} \subset\left(V^{a}\right)^{\perp}$, which is equivalent to $V^{b} \subset\left(V^{a}\right)^{\perp}$ since $V^{a} \subset V^{b}$. In other words, $\mathbf{F}^{1}$ is the zero set of the section $\omega \in H^{0}\left(\mathbb{F}(a, b, E),\left(T^{a}\right)^{*} \otimes\left(T^{b}\right)^{*}\right)$. But the bundle $\left(T^{a}\right)^{*} \otimes\left(T^{b}\right)^{*}$ is not suitable for our purpose since its rank is too big. We replace it by a subbundle of which $\omega$ is still a section. Let $\mathcal{E}$ be the kernel of the surjective morphism $\left(T^{a}\right)^{*} \otimes\left(T^{b}\right)^{*} \rightarrow S^{2}\left(T^{a}\right)^{*}$ given by the composition $\left(T^{a}\right)^{*} \otimes\left(T^{b}\right)^{*} \rightarrow$ $\left(T^{a}\right)^{*} \otimes\left(T^{a}\right)^{*} \rightarrow S^{2}\left(T^{a}\right)^{*}$. Then sections of $\left(T^{a}\right)^{*} \otimes\left(T^{b}\right)^{*}$ which come from $\Lambda^{2} E^{*}$ are sections of $\mathcal{E}$, as is the case in particular for $\omega \in \Lambda^{2} E^{*}$. The fiber of $\mathcal{E}$ at a point $\left(V^{a} \subset V^{b}\right)$ identifies, in a basis adapted to the flag $V^{a} \subset V^{b}$, to $a \times b$ matrices of the form

$$
\left(\begin{array}{ll}
A & B
\end{array}\right), \quad A \text { antisymmetric. }
$$

Such a matrix can always be completed to a $(2 n+1) \times(2 n+1)$ antisymmetric matrix

$$
\left(\begin{array}{lll}
A & B & * \\
* & * & * \\
* & * & *
\end{array}\right)
$$

which shows that $\mathcal{E}$ is generated by its global sections which come from $\Lambda^{2} E^{*}$. Since $\omega$ is generic in $\Lambda^{2} E^{*}$ and $\mathbf{F}^{1}$ is non empty, we conclude by Bertini's theorem. 
The projective variety $\mathbf{F}^{2}$ is a fibration in grassmannians $G(3 k-2(n+1), k+1)$ over the odd symplectic grassmannian $G_{\omega}(k+1, E)$, it is then smooth, irreducible, of dimension

$$
\begin{aligned}
\operatorname{dim} \mathbf{F}^{2} & =\operatorname{dim} G_{\omega}(k+1, E)+\operatorname{dim} G(3 k-2(n+1), k+1) \\
& =(k+1)(2 n-k)-\left(\begin{array}{c}
k+1 \\
2
\end{array}\right)+[3 k-2(n+1)][2(n-k)+3] .
\end{aligned}
$$

On the other hand, the variety $\mathbf{F}^{1}$ is birational to the odd symplectic grassmannian $G_{\omega}(k-1, E)$, so it is irreducible, of dimension

$$
\operatorname{dim} \mathbf{F}^{1}=\operatorname{dim} G_{\omega}(k-1, E)=(k-1)(2 n-k+2)-\left(\begin{array}{c}
k-1 \\
2
\end{array}\right) .
$$

We have then

$$
\operatorname{dim} \mathbf{F}^{2}-\operatorname{dim} \mathbf{F}^{1}=[3 k-2(n+1)][2(n-k)+1]-1
$$

so $\operatorname{dim} \mathbf{F}^{1}=\operatorname{dim} \mathbf{F}^{2}$ only when $n=k=3$, in which case $\mathbf{F}^{1}$ and $\mathbf{F}^{2}$ are both of dimension 9 .

Lemma 5.17. When $n=k=3$, the varieties $\mathbf{F}^{1}$ and $\mathbf{F}^{2}$ are not isomorphic.

Proof. We have

$$
\begin{gathered}
\mathbf{F}^{1}=\left\{\left(V^{2}, H^{5}\right) \in G_{\omega}(2,7) \times G(5,7) \mid V^{2} \subset H \subset\left(V^{2}\right)^{\perp}\right\} \\
\mathbf{F}^{2}=\left\{\left(W^{1}, V^{4}\right) \in G(1,7) \times G_{\omega}(4,7) \mid W^{1} \subset V^{4}\right\} .
\end{gathered}
$$

The variety $\mathbf{F}^{1}$ is the blow-up of $G_{\omega}(2,7)$ along the closed $\mathrm{Sp}_{7}$-orbit which is isomorphic to $G_{\omega}(1,6) \simeq \mathbb{P}^{5}$, so the exceptional divisor of $\mathbf{F}^{1}$ is a fibration in $\mathbb{P}^{3}$ over $\mathbb{P}^{5}$. The variety $\mathbf{F}^{2}$ is a fibration in $G(1,4) \simeq \mathbb{P}^{3}$ over the odd symplectic grassmannian $G_{\omega}(4,7)$. Note that the varieties $\mathbf{F}^{1}$ and $\mathbf{F}^{2}$ are both smooth of dimension 9 and of Picard group $\mathbb{Z}^{2}$. We show they don't have the same singular cohomology.

If $p: \mathbf{F}^{1} \rightarrow G_{\omega}(2,7)$ is the canonical projection and $D=p^{-1}\left(\mathbb{P}^{5}\right)$ the exceptional divisor, then, as $\mathbb{Z}$-modules, we have (cf. GH78, Ch. 4,§6])

$$
H^{*}\left(\mathbf{F}^{1}, \mathbb{Z}\right) \simeq H^{*}\left(G_{\omega}(2,7), \mathbb{Z}\right) \oplus H^{*}(D, \mathbb{Z}) / H^{*}\left(\mathbb{P}^{5}, \mathbb{Z}\right) .
$$

The $\mathbb{Z}$-module $H^{*}\left(G_{\omega}(2,7), \mathbb{Z}\right)$ is free, generated by the classes of the Schubert subvarieties of $G_{\omega}(2,7)$. It is of rank $\# \mathbf{I}_{2,7}^{\omega}=18$. Since $p: D \rightarrow \mathbb{P}^{5}$ is a fibration in $\mathbb{P}^{3}$ over $\mathbb{P}^{5}$, the ring $H^{*}(D, \mathbb{Z})$ is a free $H^{*}\left(\mathbb{P}^{5}, \mathbb{Z}\right)$-module of rank 4 (cf. GH78). As $H^{*}\left(\mathbb{P}^{5}, \mathbb{Z}\right)$ is a free $\mathbb{Z}$-module of rank 6 , we obtain that $H^{*}(D, \mathbb{Z}) / H^{*}\left(\mathbb{P}^{5}, \mathbb{Z}\right)$ is a free $\mathbb{Z}$-module of rank 18 , and so $H^{*}\left(\mathbf{F}^{1}, \mathbb{Z}\right)$ is of rank 36 .

Similarly, $\mathbf{F}^{2}$ is a fibration in $\mathbb{P}^{3}$ over $G_{\omega}(4,7)$ so $H^{*}\left(\mathbf{F}^{2}, \mathbb{Z}\right)$ is a free module of rank 4 over $H^{*}\left(G_{\omega}(4,7), \mathbb{Z}\right)$. The latter is a free $\mathbb{Z}$-module of rank $\# \mathbf{I}_{4,7}^{\omega}=8$ so $H^{*}\left(\mathbf{F}^{2}, \mathbb{Z}\right)$ is of rank 32 , and so $\mathbf{F}^{1}$ and $\mathbf{F}^{2}$ are not isomorphic.

This concludes the proof of the proposition 5.13

5.18. We can tackle now the final step in our calculation of the automorphism group of $G_{\omega}(k, E)$. Recall that what we are left to prove now is that any element of $\operatorname{Aut}\left(\mathbb{P} \Lambda^{\langle k\rangle} E, G_{\omega}(k, E)\right)$ comes from $\operatorname{PSp}_{2 n+1}$.

An element $g \in \operatorname{Aut}\left(\mathbb{P} \Lambda^{\langle k\rangle} E, G_{\omega}(k, E)\right)$ induces an automorphism of the Fano scheme $\mathbf{F}$ of [5.12 By [5.13, $\mathbf{F}$ is either irreducible and isomorphic to $\mathbf{F}^{1}$, or it has a second irreducible component $\mathbf{F}^{2}$ which is not isomorphic to $\mathbf{F}^{1}$. Therefore $g$ induces an automorphism of $\mathbf{F}^{1}$, which is to say that $g$ sends $\mathbb{P}^{2(n-k)+2}$ 's of the first kind to $\mathbb{P}^{2(n-k)+2}$ 's of the first kind.

Let $p: \mathbf{F}^{1} \rightarrow G_{\omega}(k-1, E)$ be the projection.

Proposition 5.19. The automorphism induced by $g$ on $\mathbf{F}^{1}$ permutes the fibers of $p$ and induces an automorphism $\tilde{g}$ of $G_{\omega}(k-1, E)$. 
Proof. We abuse notation and denote the automorphism induced by $g$ on $\mathbf{F}^{1}$ by the same letter $g$. The projection $p$ is an isomorphism over the open $\mathrm{Sp}_{2 n+1}$-orbit $X_{1}$ of $G_{\omega}(k-1, E)$ (cf. the proof of lemma 5.16) and the points of $p^{-1}\left(X_{1}\right)$ correspond to the $\mathbb{P}^{2(n-k)+2}$ 's of the first kind which are maximal as linear spaces contained in $G_{\omega}(k, E)$. As $g$ sends a maximal $\mathbb{P}^{2(n-k)+2}$ of the first kind on a maximal $\mathbb{P}^{2(n-k)+2}$ of the first kind, it follows that $g$ sends the open set $p^{-1}\left(X_{1}\right)$ on itself.

Let now $\left(V_{1}^{k-1}, H_{1}\right),\left(V_{2}^{k-1}, H_{2}\right)$ be two points of $p^{-1}\left(X_{0}\right) \subset \mathbf{F}^{1}$ and $L_{1}, L_{2}$ the corresponding $\mathbb{P}^{2(n-k)+2}$ 's, that is,

$$
\begin{aligned}
& L_{1}=\left\{V \in G(k, E) \mid V_{1}^{k-1} \subset V \subset H_{1}\right\} \\
& L_{2}=\left\{V \in G(k, E) \mid V_{2}^{k-1} \subset V \subset H_{2}\right\} .
\end{aligned}
$$

If $V_{1}^{k-1}=V_{2}^{k-1}=V^{k-1}$, then $H_{1} \cap H_{2}$ is of codimension 2 in $\left(V^{k-1}\right)^{\perp}$ and $L_{1}$ and $L_{2}$ cut along a $\mathbb{P}^{2(n-k)+1}$ :

$$
L_{1} \cap L_{2}=\left\{V \in G(k, E) \mid V^{k-1} \subset V \subset H_{1} \cap H_{2}\right\} \simeq \mathbb{P}^{2(n-k)+1} .
$$

If $V_{1}^{k-1} \neq V_{2}^{k-1}$ then the intersection

$$
L_{1} \cap L_{2}=\left\{V \in G(k, E) \mid V_{1}^{k-1}+V_{2}^{k-1} \subset V \subset H_{1} \cap H_{2}\right\}
$$

is either empty or it reduces to a point: if $V \in L_{1} \cap L_{2}$ then necessarily $V=$ $V_{1}^{k-1}+V_{2}^{k-1}$ since $V_{1}^{k-1}$ and $V_{2}^{k-1}$ are distinct hyperplanes in $V$. It follows that $g$ sends a fiber of $p$ on a fiber of $p$, and so it induces a bijection $\tilde{g}$ such that the following square commutes:

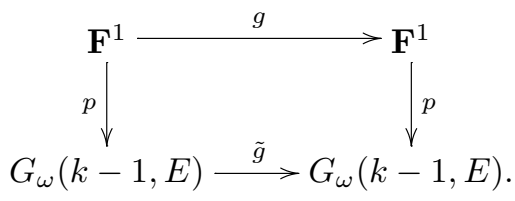

Since $\mathbf{F}^{1}$ is projective, the projection $p$ is a closed map and so $\tilde{g}$ is continuous for the Zariski topology. On the other hand, $\tilde{g}$ is regular on the open orbit $X_{1}$ since $p$ is an isomorphism over $X_{1}$. The closed orbit $X_{0}$ is of codimension at least 2 , and since $G_{\omega}(k-1, E)$ is normal this implies that $\tilde{g}$ is regular everywhere. Indeed, for any affine open set $U \subset G_{\omega}(k-1, E)$ the preimage $\tilde{g}^{-1}(U)$ is normal and the restriction $\left.\tilde{g}\right|_{\tilde{g}^{-1}(U)}$ is given by some rational functions, which are regular outside a codimension 2 closed set and therefore extend to the whole of $\tilde{g}^{-1}(U)$. It follows that $\left.\tilde{g}\right|_{X_{1}}$ extends to a regular map on $G_{\omega}(k-1, E)$ which, by continuity, has to be $\tilde{g}$.

We have just shown that an automorphism of $G_{\omega}(k, E)$ induces an automorphism of $G_{\omega}(k-1, E)$. Iterating, it follows that an automorphism of $G_{\omega}(k, E)$ induces an automorphism of $G_{\omega}(1, E)=\mathbb{P} E$, that is, an element $h \in \operatorname{PSL}(E)$. We now only need to show that $h$ is in $\mathrm{PSp}_{2 n+1}$ and that the automorphism of $G_{\omega}(k, E)$ it defines coincides with our initial automorphism. This is done in the following two lemmas.

Lemma 5.20. If the automorphism of $G(2, E)$ induced by some $h \in \operatorname{PSL}(E)$ preserves $G_{\omega}(2, E)$ then $h \in \mathrm{PSp}_{2 n+1}$.

Proof. Let $\hat{h}$ be a representative of $h$ in $\operatorname{SL}(E)$. Then $\hat{h}$ preserves orthogonality, that is, for all $u, v \in E$

$$
u \perp v \Rightarrow \hat{h} u \perp \hat{h} v,
$$

since an isotropic subspace of dimension 2 is generated by two orthogonal vectors and conversely. In other words, the automorphism $(u, v) \mapsto(\hat{h} u, \hat{h} v)$ preserves the 
affine closed set

$$
\{(u, v) \in E \times E \mid \omega(u, v)=0\} .
$$

Since $\omega$ is irreducible as a degree two polynomial on $E \times E$, it follows that there is $\lambda \in \mathbb{C}^{*}$ such that $\omega(\hat{h} u, \hat{h} v)=\lambda \omega(u, v)$ for all $u, v \in E$, ie that $\hat{h} \omega=\lambda^{-1} \omega$. So, if $\mu \in \mathbb{C}^{*}$ is a square root of $\lambda^{-1}$, then $(\mu \hat{h}) \omega=\omega$, that is, $\mu \hat{h} \in \operatorname{Sp}_{2 n+1}$ and so $h \in \mathrm{PSp}_{2 n+1}$.

Lemma 5.21. Let $g$ be an automorphism of $G_{\omega}(k, E)$ and $\tilde{g}$ the automorphism it induces on $G_{\omega}(k-1, E)$. If $\tilde{g}$ is induced by some $h \in \mathrm{PSp}_{2 n+1}$ then $g$ is also induced by $h$.

Proof. Through any point $V \in G_{\omega}(k, E)$ passes a $\mathbb{P}^{2(n-k)+2}$ of the first kind. Indeed, it suffices to choose $V^{k-1} \subset V$ of dimension $k-1$ and $H$ of dimension $2 n-k+2$ such that $V \subset H \subset\left(V^{k-1}\right)^{\perp}$, and to consider the linear space $L$ they define by (13). Then $g L$ passes through $g V$ and the commuting square (15) shows then that for all $V \in G_{\omega}(k, E)$ and $V^{k-1} \in G(k-1, E)$ we have

$$
V^{k-1} \subset V \Longrightarrow \tilde{g} V^{k-1}=h\left(V^{k-1}\right) \subset g V .
$$

Since the hyperplane $V^{k-1} \subset V$ is arbitrary, it follows that $g V=h(V)$.

\section{A Borel-WeIL THEOREM FOR THE ODD SYMPLECTIC GROUP}

We set out to prove the Borel-Weil type theorem stated in the introduction. We start by examining the preferred classes of modules which appear as the spaces of global sections of line bundles on the odd symplectic flag manifold $\mathbb{F}_{\omega}(2 n+1)$.

6.1. Shtepin's class of $\widetilde{\mathfrak{s p}}_{2 n+1}$-modules. In [Sht93], Shtepin deals with the problem of separating multiple components when restricting simple $\mathfrak{s p}_{2 n+2}$-modules to $\mathfrak{s p}_{2 n}$. There is no semi-simple Lie algebra sitting between $\mathfrak{s p}_{2 n+2}$ and $\mathfrak{s p}_{2 n}$ which could be used as an intermediate step in the reduction $\mathfrak{s p}_{2 n+2} \downarrow \mathfrak{s p}_{2 n}$. Instead, Shtepin considers the non-reductive intermediate Lie algebra $\widetilde{\mathfrak{s p}}_{2 n+1}$ and constructs in each simple $\mathfrak{s p}_{2 n+2}$-module $V$ a filtration by $\widetilde{\mathfrak{s p}}_{2 n+1}$-modules

$$
V_{1} \subset \cdots \subset V_{p} \subset V
$$

such that the factors $V_{i+1} / V_{i}$ are pairwise non-isomorphic and multiplicity free $\mathfrak{s p}_{2 n}$-modules. It turns out that the $\widetilde{\mathfrak{s p}}_{2 n+1}$-modules $V_{i+1} / V_{i}$ have nice properties, in particular as we will show below, they appear as spaces of global sections of line bundles on $\mathbb{F}_{\omega}(2 n+1)$. We now give a short account of this construction.

We will use Shtepin's definition for the intermediate Lie algebra, which is

$$
\widetilde{\mathfrak{s p}}_{2 n+1}=\left\{X \in \mathfrak{s p}_{2 n+2} \mid X e_{n}=0\right\} .
$$

This is not just a mere change of notation with respect to our definition from 3.11 since we still want to distinguish the Borel subalgebra $\mathfrak{b}_{2 n+2}$ of $\mathfrak{s p}_{2 n+2}$ of upper triangular matrices in the symplectic basis $\left\{e_{0}, \ldots, e_{2 n+1}\right\}$ as giving the positive roots and thus the notion of dominant and highest weights. Of course, we can get back to our definition from 3.11 by conjugating by some element $g \in \mathrm{Sp}_{2 n+2}$ which sends $e_{n}$ to $e_{0}$, but then we will also have to conjugate the Borel subalgebra and consider as positive roots the roots of $g \mathfrak{b}_{2 n+2} g^{-1}$, which is rather awkward. In accordance with the definition (16) we now embed $\mathfrak{s p}_{2 n} \subset \mathfrak{s p}_{2 n+2}$ as the subalgebra

$$
\mathfrak{s p}_{2 n}=\left\{X \in \mathfrak{s p}_{2 n+2} \mid X e_{n}=0, X e_{\bar{n}}=0\right\}
$$

Let $\mathfrak{t}_{2 n+2}$ be the Cartan subalgebra of $\mathfrak{s p}_{2 n+2}$ of diagonal matrices in the symplectic basis $\left\{e_{0}, \ldots, e_{2 n+1}\right\}$ and $\mathfrak{b}_{2 n+2}^{-}$the Borel subalgebra opposite to $\mathfrak{b}_{2 n+2}$.

Denote $\mathfrak{u}_{2 n+2}^{-}, \mathfrak{u}_{2 n+2}^{+}$the maximal nilpotent subalgebras of $\mathfrak{b}_{2 n+2}^{-}$and $\mathfrak{b}_{2 n+2}$. Let $\mathfrak{t}_{2 n}=\mathfrak{t}_{2 n+2} \cap \mathfrak{s p}_{2 n}, \mathfrak{b}_{2 n}=\mathfrak{b}_{2 n+2} \cap \mathfrak{s p}_{2 n}$, respectively $\mathfrak{u}_{2 n}^{ \pm}=\mathfrak{u}_{2 n+2}^{ \pm} \cap \mathfrak{s p}_{2 n}$ be the 
corresponding subalgebras of $\mathfrak{s p}_{2 n}$. Denote also $\mathfrak{u}_{2 n+1}^{ \pm}=\mathfrak{u}_{2 n+2}^{ \pm} \cap \widetilde{\mathfrak{p}}_{2 n+1}$ and $\mathfrak{b}_{2 n+1}=\mathfrak{b}_{2 n+2} \cap \widetilde{\mathfrak{s p}}_{2 n+1}$. Then $\mathfrak{b}_{2 n+1}=\mathfrak{t}_{2 n} \oplus \mathfrak{u}_{2 n+1}^{+}$, but note that $\mathfrak{b}_{2 n+1}$ is not a Borel subalgebra of $\widetilde{s p}_{2 n+1}$. We have the decomposition

$$
\widetilde{\mathfrak{s}}_{2 n+1}=\mathfrak{u}_{2 n+1}^{-} \oplus \mathfrak{t}_{2 n} \oplus \mathfrak{u}_{2 n+1}^{+} .
$$

Note that since we have

$$
\begin{aligned}
\mathfrak{u}_{2 n+1}^{+} & =\mathfrak{u}_{2 n}^{+} \oplus \bigoplus_{0 \leqslant i \leqslant n-1} \mathbb{C} X_{i \bar{n}} \oplus \mathbb{C} X_{n \bar{n}} \\
\mathfrak{u}_{2 n+1}^{-} & =\mathfrak{u}_{2 n}^{-} \oplus \bigoplus_{0 \leqslant i \leqslant n-1} \mathbb{C} X_{n i}
\end{aligned}
$$

the $\mathfrak{t}_{2 n}$-weights of $\mathfrak{u}_{2 n+1}^{+}$are the positive roots of $\mathfrak{s p}_{2 n}$ to which we add the $\varepsilon_{i}$, for $1 \leqslant i \leqslant n-1$ (and 0 corresponding to the element $X_{n \bar{n}}$ ) and the $\mathfrak{t}_{2 n}$-weights of $\mathfrak{u}_{2 n+1}^{-}$are their negatives (except for the 0 weight which does not appear). Having this analogy between the decomposition (17) and a Cartan decomposition is the reason to choosing the definition (16) instead of our earlier one.

Let $V$ be a simple $\mathfrak{s p}_{2 n+2}$-module. Shtepin constructs his filtration in $V$ using an explicit model of $V$ as a space of functions on the unipotent group $U_{2 n+2}^{+}$with Lie algebra $\mathfrak{u}_{2 n+2}^{+}$due to Želobenko (cf. ZZel62]), which we won't detail here. Following Shtepin, we call semi-maximal the $\mathfrak{t}_{2 n+2}$-eigenvectors of $V$ which are killed by $\mathfrak{u}_{2 n}^{+}$. These are exactly the highest weight vectors of the simple pieces of a decomposition of $V$ as a $\mathfrak{s p}_{2 n}$-module. Among the semi-maximal vectors we choose those which are killed by the elements $X_{0 n}, X_{1 n}, \ldots, X_{n-1 n}$ of $\mathfrak{u}_{2 n+2}^{+}$, which we call quasi-maximal. We order them $v_{1}, \ldots, v_{p}$ in a natural way coming from their explicit expressions in the Želobenko model and we define then the filtration $V_{1} \subset \cdots \subset V_{p}$ in $V$ by taking $V_{i}$ to be the $\widetilde{\mathfrak{s p}}_{2 n+1}$-module generated by $v_{1}, \ldots, v_{i}$.

The ordering $v_{1}, \ldots, v_{p}$ is chosen such that for all $1 \leqslant i \leqslant p-1$ we have $\mathfrak{u}_{2 n+1}^{+} v_{i+1} \in V_{i}$, so the image $\bar{v}_{i+1}$ of $v_{i+1}$ in $V_{i+1} / V_{i}$ is a vector killed by $\mathfrak{u}_{2 n+1}^{+}$, ie a maximal vector with respect to $\widetilde{\mathfrak{s p}}_{2 n+1}$. The factor $V_{i+1} / V_{i}$ is a cyclic $\widetilde{\mathfrak{s p}}_{2 n+1^{-}}$ module generated by the maximal vector $\bar{v}_{i+1}$, and so, if $\mu$ is the $\mathfrak{t}_{2 n}$-weight of $\bar{v}_{i+1}$, $V_{i+1} / V_{i}$ is a quotient of the Verma-like module

$$
U\left(\widetilde{\mathfrak{s p}}_{2 n+1}\right) \otimes_{U\left(\mathfrak{b}_{2 n+1}\right)} \mathbb{C}_{\mu}
$$

where $\mathbb{C}_{\mu}$ is the $\mathfrak{b}_{2 n+1}$-module of dimension 1 on which $\mathfrak{b}_{2 n+1}$ acts through the weight $\mu$. This already accounts for some properties of the $\widetilde{\mathfrak{s p}}_{2 n+1}$-module $V_{i+1} / V_{i}$ similar to properties of simple modules for semi-simple Lie algebras, such as:

(1) $\mu$ is the highest weight of $V_{i+1} / V_{i}$, ie the weights of $V_{i+1} / V_{i}$ are of the form $\mu-\theta$ with $\theta$ in the semi-group generated by the positive roots of $\widetilde{\mathfrak{s p}}_{2 n+1}$;

(2) the Weyl group $W\left(\mathrm{Sp}_{2 n}\right)$ acts on the weights of $V_{i+1} / V_{i}$ and for any $w \in$ $W\left(\mathrm{Sp}_{2 n}\right)$ the $w \mu$-eigenspace is of dimension 1 ;

(3) any $\widetilde{\mathfrak{s p}}_{2 n+1}$-module endomorphism of $V_{i+1} / V_{i}$ is scalar, in particular $V_{i+1} / V_{i}$ is indecomposable;

(4) $V_{i+1} / V_{i}$ has a lowest weight, which is $\bar{w}_{0} \mu$ where $\bar{w}_{0}$ is the longest element of $W\left(\mathrm{Sp}_{2 n}\right)$;

etc.

Actually, if $\lambda$ is the highest weight of the simple $\mathfrak{s p}_{2 n+2}$-module $V$, then the quasimaximal vectors of $V$ (and so the factors $V_{i+1} / V_{i}$ ) are parametrized by patterns $\mu \rightarrow \lambda$, where $\mu$ is also an $\mathfrak{s p}_{2 n+2}$-dominant weight and where the notation $\mu \rightarrow \lambda$ means

$$
\lambda_{1} \geqslant \mu_{1} \geqslant \lambda_{2} \geqslant \cdots \geqslant \mu_{n} \geqslant \lambda_{n+1} \geqslant \mu_{n+1} .
$$


The quasimaximal vector $q_{\mu, \lambda}$ corresponding to the pattern $\mu \rightarrow \lambda$ is a $\mathfrak{t}_{2 n+2^{-}}$ eigenvector with weight

$$
\mu-(|\lambda|-|\mu|) \varepsilon_{n},
$$

in particular its $\mathfrak{t}_{2 n}$-weight is $\left.\mu\right|_{\mathfrak{t}_{2 n}}$. Let us denote by $V_{\lambda}$ the simple $\mathfrak{s p}_{2 n+2}$-module with highest weight $\lambda$. Shtepin proves then the following result:

Theorem 6.2. The factor of the filtration of $V_{\lambda}$ generated by the image of the quasimaximal vector $q_{\mu, \lambda}$ is isomorphic to the $\widetilde{\mathfrak{s p}}_{2 n+1}$-submodule of $V_{\mu}$ generated by the highest weight vector.

Denote by $V_{\mu}^{\prime}$ this $\widetilde{\mathfrak{s p}}_{2 n+1}$-module. Note that $V_{\mu}^{\prime}$ does not depend only on $\left.\mu\right|_{\mathfrak{t}_{2 n}}$, as one might suspect given that it is a quotient of the Verma module (18). In fact it is already so for its structure as an $\mathfrak{s p}_{2 n}$-module, as Shtepin shows that the decomposition of $V_{\mu}^{\prime}$ into irreducible $\mathfrak{s p}_{2 n}$-modules is

$$
\bigoplus_{\nu \rightarrow \mu} V_{\nu}^{\prime \prime}
$$

where $V_{\nu}^{\prime \prime}$ is the simple $\mathfrak{s p}_{2 n}$-module of highest weight $\nu$ and where the notation $\nu \rightarrow \mu$ now means

$$
\mu_{1} \geqslant \nu_{1} \geqslant \mu_{2} \geqslant \cdots \geqslant \mu_{n} \geqslant \nu_{n} \geqslant \mu_{n+1} .
$$

We will also need to consider the parabolic subalgebra

$$
\mathfrak{p}=\left\{X \in \mathfrak{s p}_{2 n+2} \mid X\left(\mathbb{C} e_{n}\right) \subset \mathbb{C} e_{n}\right\} .
$$

We have

$$
\mathfrak{p}=\widetilde{\mathfrak{s p}}_{2 n+1} \oplus \mathbb{C} X_{n n}
$$

and since $X_{n n} \in \mathfrak{t}_{2 n+2}$, the modules $V_{i}$ of the filtration of a simple $\mathfrak{s p}_{2 n+2}$-module $V$ are also $\mathfrak{p}$-modules. Similarly, the $\widetilde{\mathfrak{s p}}_{2 n+1}$-submodule $V_{\mu}^{\prime}$ of a simple $\mathfrak{s p}_{2 n+2}$-module $V_{\mu}$ generated by the highest weight vector is a $\mathfrak{p}$-module. Then from the theorem 6.2 it follows that the factor of the filtration of a simple $\mathfrak{s p}_{2 n+2}$-module $V_{\lambda}$ generated by the image of the quasimaximal vector $q_{\mu, \lambda}$ is isomorphic to the $\mathfrak{p}$-module

$$
V_{\mu}^{\prime} \otimes \mathbb{C}_{-(|\lambda|-|\mu|) \varepsilon_{n}} .
$$

6.3. The $\mathfrak{s p}_{2 n+1}$-modules of Proctor. We continue to use the definition (16) and accordingly, by $\mathfrak{s p}_{2 n+1}$ we mean the Lie algebra of the odd symplectic group $\operatorname{Sp}_{2 n+1}$ associated to the odd symplectic form $\left.\omega\right|_{e_{n}^{+}}$. Recall from 3.6 that the restriction morphism $\left.g \mapsto g\right|_{e_{n}^{\perp}}$ induces a surjective morphism $\mathfrak{p} \rightarrow \mathfrak{s p}_{2 n+1}$ which restricts to a surjective morphism $\widetilde{\mathfrak{s p}}_{2 n+1} \rightarrow \mathfrak{s p}_{2 n+1} \cap \mathfrak{s l}_{2 n+1}$. The kernel of both these morphisms is the one dimensional space $\mathbb{C} X_{n \bar{n}}$.

In [Pro88, Proctor uses an adapted form of the construction of Weyl to define a special class of $\mathfrak{s p}_{2 n+1}$-modules. Let us recall this construction.

Let $\omega \in \Lambda^{2} V^{*}$ be a generic skew-form on a complex vector space $V$, and let $\lambda$ be a partition. The GL $(V)$-module of highest weight $\lambda$ coincides with the Schur power $S_{\lambda} V \subset V^{\otimes d}$, where $d=|\lambda|$. For $1 \leqslant p<q \leqslant d$, denote $\varphi_{p q}$ the contraction by $\omega$ on the indices $p$ et $q$

$$
\begin{gathered}
\varphi_{p q}: V^{\otimes d} \longrightarrow V^{\otimes d-2} \\
v_{1} \otimes \cdots \otimes v_{n} \longmapsto \omega\left(v_{p}, v_{q}\right) v_{1} \otimes \cdots \otimes \widehat{v_{p}} \otimes \cdots \otimes \widehat{v_{q}} \otimes \cdots \otimes v_{n} .
\end{gathered}
$$

Following [FH91] §17.3], denote

$$
V^{\langle d\rangle}=\bigcap_{p, q} \operatorname{Ker} \varphi_{p q} \subset V^{\otimes d}
$$

the space of "trace free" $d$-tensors, and finally

$$
S_{\langle\lambda\rangle} V=S_{\lambda} V \cap V^{\langle d\rangle} .
$$


When $V=\mathbb{C}^{2 n}$, if $\ell(\lambda)>n$ then $S_{\langle\lambda\rangle} \mathbb{C}^{2 n}=0$, and if $\ell(\lambda) \leqslant n$ then $S_{\langle\lambda\rangle} \mathbb{C}^{2 n}$ is the simple $\mathfrak{s p}_{2 n}$-module of highest weight $\lambda$.

In Pro88, Proctor studies the $\mathfrak{s p}_{2 n+1}$-modules $S_{\langle\lambda\rangle} \mathbb{C}^{2 n+1}$. He shows for example the following (cf. Pro88 theorem 2.1]) :

Proposition 6.4. If $\ell(\lambda)>n+1$ then $S_{\langle\lambda\rangle} \mathbb{C}^{2 n+1}=0$. If $\ell(\lambda) \leqslant n+1$ then the $\mathfrak{s p}_{2 n+1}$-module $S_{\langle\lambda\rangle} \mathbb{C}^{2 n+1}$ is indecomposable.

He further describes weight bases for the modules $S_{\langle\lambda\rangle} \mathbb{C}^{2 n+1}$ and gives a formula for the $\mathfrak{t}_{2 n}$-character of $S_{\langle\lambda\rangle} \mathbb{C}^{2 n+1}$ similar to Weyl's formula. As it turns out (cf. Pro88 corollary 8.1]), the decomposition of $S_{\langle\lambda\rangle} \mathbb{C}^{2 n+1}$ in simple $\mathfrak{s p}_{2 n}$-components is also given by the formula (19). This is not a mere coincidence, since we have:

Proposition 6.5. As a $\mathfrak{p - m o d u l e ~ v i a ~ t h e ~ m o r p h i s m ~} \mathfrak{p} \rightarrow \mathfrak{s p}_{2 n+1}, S_{\langle\lambda\rangle} \mathbb{C}^{2 n+1}$ is isomorphic to the $\mathfrak{p}$-module $V_{\lambda}^{\prime}$.

Proof. Let $\tilde{E}=\mathbb{C}^{2 n+2}$ and $\tilde{\omega} \in \Lambda^{2} \tilde{E}^{*}$ be the symplectic form on $\tilde{E}$ for which the basis $\left\{e_{0}, \ldots, e_{2 n+1}\right\}$ is symplectic. Denote $E=e_{n}^{\perp}$ and $\omega=\left.\tilde{\omega}\right|_{E}$. We identify the simple $\mathfrak{s p}_{2 n+2}$-module $V_{\lambda}$ of highest weight $\lambda$ with $S_{\langle\lambda\rangle} \tilde{E}$. The highest weight vector $v_{\lambda} \in V_{\lambda}$ coincides in this case with the image in $S_{\lambda} \tilde{E}$ of the tensor

$$
e^{\lambda}=e_{0}^{\otimes \lambda_{0}} \otimes e_{1}^{\otimes \lambda_{1}} \otimes \cdots \otimes e_{n}^{\otimes \lambda_{n}} \in \tilde{E}^{\otimes|\lambda|} .
$$

But since $e^{\lambda} \in E^{\otimes|\lambda|}$, we have $v_{\lambda} \in S_{\lambda} E \subset S_{\lambda} \tilde{E}$. On the other hand, $v_{\lambda} \in E^{\langle|\lambda|\rangle}$ since clearly the contractions (20) defined by $\omega$ are just the restrictions to $E^{\otimes|\lambda|}$ of the contractions (20) defined by $\tilde{\omega}$. Therefore $v_{\lambda} \in S_{\langle\lambda\rangle} E$.

Since $\mathfrak{p}$ acts on $S_{\langle\lambda\rangle} E$ through the restriction morphism $\mathfrak{p} \rightarrow \mathfrak{s p}_{2 n+1}$, it follows that the p-module $V_{\lambda}^{\prime}$ generated by $v_{\lambda}$ is actually contained in $S_{\langle\lambda\rangle} E$. But $V_{\lambda}^{\prime}$ and $S_{\langle\lambda\rangle} E$ have the same dimension, since they are isomorphic as $\mathfrak{s p}_{2 n}$-modules. Therefore they must coincide.

6.6. Borel-Weil for the odd symplectic group. Let $G$ be a semi-simple complex Lie group, $B \subset G$ a Borel subgroup and $T \subset B$ a maximal torus. Denote $W$ the associated Weyl group. Let $e^{1}$ be the fixed point in the flag variety $G / B$ of the opposite Borel subgroup $B^{-}$. Denote then, for $w \in W, e^{w}=w\left(e^{1}\right)$ and $X^{w}$ the opposite Schubert variety which is the closure of the $B^{-}$-orbit of $e^{w}$. For a weight $\lambda$ let $L_{\lambda}$ be the line bundle on $G / B$ whose fiber at the point $e^{1}$ is the $B^{-}$-module $\mathbb{C}_{\lambda}$. By the Borel-Weil theorem, if $\lambda$ is dominant then $H^{0}\left(G / B, L_{\lambda}\right) \simeq V_{\lambda}$, where $V_{\lambda}$ is the simple $G$-module of highest weight $\lambda$. Denote the restriction of $L_{\lambda}$ to the Schubert variety $X^{w}$ also by $L_{\lambda}$. Then (cf. Jan03 §14.19]) the restriction morphism

$$
H^{0}\left(G / B, L_{\lambda}\right) \longrightarrow H^{0}\left(X^{w}, L_{\lambda}\right)
$$

is surjective and

$$
H^{0}\left(X^{w}, L_{\lambda}\right)^{*} \simeq \operatorname{Span}\left(B^{-} \cdot v_{-w \lambda}\right) \subset V_{\lambda}^{*}
$$

where $v_{-w \lambda} \in V_{\lambda}^{*}$ is a vector of weight $-w \lambda$ (unique up to a scalar factor) and $\operatorname{Span}\left(B^{-} \cdot v_{-w \lambda}\right)$ is the $B^{-}$-submodule it generates.

Denote also $e_{1}$ the fixed point in $G / B$ of $B$, and, for $w \in W, X_{w}$ the Schubert variety which is the closure of the $B$-orbit of $e_{w}=w\left(e_{1}\right)$. Then $X_{w}=w_{0} X^{w}$, where $w_{0}$ is the longest element of $W$. Conjugating by $w_{0}$ in (21) we get

$$
H^{0}\left(X_{w}, L_{\lambda}\right)^{*} \simeq \operatorname{Span}\left(B \cdot v_{-w_{0} w \lambda}\right) \subset V_{\lambda}^{*} .
$$

Take now $G=\mathrm{Sp}_{2 n+2}, B_{2 n+2} \subset \mathrm{Sp}_{2 n+2}$ the Borel subgroup of upper triangular matrices in the symplectic basis $\left\{e_{0}, \ldots, e_{2 n+1}\right\}, T_{2 n+2} \subset B_{2 n+2}$ the maximal torus of diagonal matrices in the basis $\left\{e_{0}, \ldots, e_{2 n+1}\right\}$, and denote $E=e_{0}^{\perp}$. Let $w=$ $\overline{1} \overline{2} \ldots \bar{n} 0 \in W\left(\mathrm{Sp}_{2 n+2}\right)$ so that, as $\operatorname{in} 4.17 \mathbb{F}_{\omega}(E)=X_{\overline{1} \overline{2} \ldots \bar{n} 0}$. Note that the longest 
element $w_{0}$ of the Weyl group $W\left(\mathrm{Sp}_{2 n+2}\right)$ acts as -1 and $V_{\lambda}^{*} \simeq V_{\lambda}$. From (22) we get

$$
H^{0}\left(\mathbb{F}_{\omega}(E), L_{\lambda}\right)^{*} \simeq \operatorname{Span}\left(B_{2 n+2} \cdot v_{w \lambda}\right) \subset V_{\lambda} .
$$

Since $\mathbb{F}_{\omega}(E)$ is $P$-stable, where $P$ is the parabolic subgroup preserving the line $\mathbb{C e}_{0}$, we have $\operatorname{Span}\left(B_{2 n+2} \cdot v_{w \lambda}\right)=\operatorname{Span}\left(P \cdot v_{w \lambda}\right)$ and this isomorphism is also an isomorphism of $P$-modules.

Denote also $E^{\prime}=e_{n}^{\perp}$ and consider $\mathbb{F}_{\omega}\left(E^{\prime}\right)$ which embeds in $\mathbb{F}_{\omega}(2 n+2)$ as

$$
\left\{\left(V_{1} \subset \cdots \subset V_{n+1}\right) \in \mathbb{F}_{\omega}(2 n+2) \mid V_{n+1} \subset E^{\prime}\right\} .
$$

Let $P^{\prime}$ be the parabolic subgroup preserving the line $\mathbb{C} e_{n}$ and $\mathfrak{p}^{\prime}$ its Lie algebra (which in 6.1 has been denoted $\mathfrak{p}$ ).

Proposition 6.7. As a $\mathfrak{p}^{\prime}$-module, $H^{0}\left(\mathbb{F}_{\omega}\left(E^{\prime}\right), L_{\lambda}\right)^{*}$ is isomorphic to Shtepin's $V_{\lambda}^{\prime}$.

Proof. Since $w(n)=0$, we have $\mathbb{F}_{\omega}\left(E^{\prime}\right)=w^{-1} \mathbb{F}_{\omega}(E)$ and $P^{\prime}=w^{-1} P w$, so conjugating by $w$ in 23], we get $H^{0}\left(\mathbb{F}_{\omega}\left(E^{\prime}\right), L_{\lambda}\right)^{*} \simeq \operatorname{Span}\left(P^{\prime} \cdot v_{\lambda}\right)=V_{\lambda}^{\prime}$.

Corollary 6.8. As $\mathfrak{p}^{\prime}$-modules, $H^{0}\left(\mathbb{F}_{\omega}\left(E^{\prime}\right), L_{\lambda}\right)^{*} \simeq S_{\langle\lambda\rangle} E^{\prime}$.

Proof. This follows immediately from 6.5

Let now $T_{i}$ be the tautological bundle of rank $i$ on the symplectic flag manifold $\mathbb{F}_{\omega}(2 n+2)$, whose fiber at a point $\left(V_{1} \subset \cdots \subset V_{n+1}\right)$ is $V_{i}$. The restriction $\left.T_{i}\right|_{\mathbb{F}_{\omega}(E)}$ is then the rank $i$ tautological bundle on $\mathbb{F}_{\omega}(E)$ and we denote it also by $T_{i}$. We have $T_{i+1} / T_{i}=L_{-\varepsilon_{i}}$ for $1 \leqslant i \leqslant n$ and $T_{1}=L_{-\varepsilon_{0}}$ so

$$
L_{\lambda}=T_{1}^{* \otimes \lambda_{0}} \otimes\left(T_{2} / T_{1}\right)^{* \otimes \lambda_{1}} \otimes \cdots \otimes\left(T_{n+1} / T_{n}\right)^{* \otimes \lambda_{n}} .
$$

Theorem 6.9. Let $\lambda=\left(\lambda_{0} \geqslant \cdots \geqslant \lambda_{n} \geqslant 0\right)$ be a partition and $L_{\lambda}$ the line bundle on $\mathbb{F}_{\omega}(E)$

$$
L_{\lambda}=T_{1}^{* \otimes \lambda_{0}} \otimes\left(T_{2} / T_{1}\right)^{* \otimes \lambda_{1}} \otimes \cdots \otimes\left(T_{n+1} / T_{n}\right)^{* \otimes \lambda_{n}} .
$$

Then as $\mathrm{Sp}_{2 n+1}$-modules, $H^{0}\left(\mathbb{F}_{\omega}(E), L_{\lambda}\right)^{*} \simeq S_{\langle\lambda\rangle} E$.

Proof. First denote $\mathrm{Sp}_{2 n+1}^{\prime}$ the odd symplectic group which is the image of $P^{\prime}$ via the restriction morphism $\left.g \mapsto g\right|_{E^{\prime}}$. Then the modules in 6.8 are also $\mathrm{Sp}_{2 n+1^{-}}^{\prime}$ modules. We have $\mathrm{Sp}_{2 n+1}=w \mathrm{Sp}_{2 n+1}^{\prime} w^{-1}$ and the result follows from 6.8 once we observe that $S_{\langle\lambda\rangle} E=w S_{\langle\lambda\rangle} E^{\prime}$ as subspaces of $S_{\langle\lambda\rangle} \mathbb{C}^{2 n+2}$.

Note that the statement of theorem 6.9 is independent of the embedding $\mathbb{F}_{\omega}(E) \subset$ $\mathbb{F}_{\omega}(2 n+2)$.

\section{REFERENCES}

[Akh95] D.N. Akhiezer, Lie group actions in complex analysis, Aspects of Mathematics, E27, Friedr. Vieweg \& Sohn, Braunschweig, 1995.

[Bou68] N. Bourbaki, Éléments de mathématique. Fasc. XXXIV. Groupes et algèbres de Lie. Chapitre IV: Groupes de Coxeter et systèmes de Tits. Chapitre V: Groupes engendrés par des réflexions. Chapitre VI: systèmes de racines, Actualités Scientifiques et Industrielles, No. 1337, Hermann, Paris, 1968.

[FH91] W. Fulton and J. Harris, Representation theory, Graduate Texts in Mathematics, vol. 129, Springer-Verlag, New York, 1991, A first course, Readings in Mathematics.

[GH78] Ph. Griffiths and J. Harris, Principles of algebraic geometry, Wiley-Interscience [John Wiley \& Sons], New York, 1978, Pure and Applied Mathematics.

[GZ84] I.M. Gel'fand and A.V. Zelevinskǐ̌, Models of representations of classical groups and their hidden symmetries, Funktsional. Anal. i Prilozhen. 18 (1984), no. 3, 14-31.

[Har77] R. Hartshorne, Algebraic geometry, Springer-Verlag, New York, 1977, Graduate Texts in Mathematics, No. 52.

[Har95] J. Harris, Algebraic geometry, Graduate Texts in Mathematics, vol. 133, Springer-Verlag, New York, 1995, A first course, Corrected reprint of the 1992 original. 
[Jan03] J.C. Jantzen, Representations of algebraic groups, second ed., Mathematical Surveys and Monographs, vol. 107, American Mathematical Society, Providence, RI, 2003.

[LM06] J.M. Landsberg and L. Manivel, The sextonions and $\mathrm{E}_{7 \frac{1}{2}}$, Adv. Math. 201 (2006), no. 1, 143-179.

[Mac95] I.G. Macdonald, Symmetric functions and Hall polynomials, second ed., Oxford Mathematical Monographs, The Clarendon Press Oxford University Press, New York, 1995, With contributions by A. Zelevinsky, Oxford Science Publications.

[Mih] I.A. Mihai, Equivariant cohomology of the odd symplectic flag manifold, in preparation.

[Mih05] _ Variétés de drapeaux symplectiques impaires, Ph.D. thesis, Institut Fourier, 2005.

[Pro82] R.A. Proctor, Classical Bruhat orders and lexicographic shellability, J. Algebra 77 (1982), no. $1,104-126$

[Pro88] - Odd symplectic groups, Invent. Math. 92 (1988), no. 2, 307-332.

[Sht93] V.V. Shtepin, Intermediate Lie algebras and their finite-dimensional representations, Izv. Ross. Akad. Nauk Ser. Mat. 57 (1993), no. 6, 176-198

[Žel62] D.P. Želobenko, Classical groups. Spectral analysis of finite-dimensional representations, Uspehi Mat. Nauk 17 (1962), no. 1 (103), 27-120. 